\title{
Material Generation Algorithm: A Novel Metaheuristic Algorithm for Optimization of Engineering Problems
}

\author{
Siamak Talatahari ${ }^{1,2}\left(\mathbb{D}\right.$, Mahdi Azizi $^{1}(\mathbb{D})$ and Amir H. Gandomi ${ }^{3, *}(\mathbb{C}$ \\ 1 Department of Civil Engineering, University of Tabriz, Tabriz 5166616471, Iran; siamak.talat@gmail.com (S.T.); \\ mehdi.azizi875@gmail.com (M.A.) \\ 2 Engineering Faculty, Near East University, North Cyprus, Mersin 10, Turkey \\ 3 Faculty of Engineering \& Information Technology, University of Technology Sydney, Ultimo, \\ Sydney, NSW 2007, Australia \\ * Correspondence: gandomi@uts.edu.au
}

Citation: Talatahari, S.; Azizi, M.; Gandomi, A.H. Material Generation Algorithm: A Novel Metaheuristic Algorithm for Optimization of Engineering Problems. Processes 2021, 9, 859. https://doi.org/10.3390/ pr9050859

Academic Editors: Luis Puigjaner and Ján Pitel'

Received: 30 March 2021

Accepted: 10 May 2021

Published: 13 May 2021

Publisher's Note: MDPI stays neutral with regard to jurisdictional claims in published maps and institutional affiliations.

Copyright: (c) 2021 by the authors. Licensee MDPI, Basel, Switzerland. This article is an open access article distributed under the terms and conditions of the Creative Commons Attribution (CC BY) license (https:// creativecommons.org/licenses/by/ $4.0 /)$.

\begin{abstract}
A new algorithm, Material Generation Algorithm (MGA), was developed and applied for the optimum design of engineering problems. Some advanced and basic aspects of material chemistry, specifically the configuration of chemical compounds and chemical reactions in producing new materials, are determined as inspirational concepts of the MGA. For numerical investigations purposes, 10 constrained optimization problems in different dimensions of 10, 30, 50, and 100, which have been benchmarked by the Competitions on Evolutionary Computation (CEC), are selected as test examples while 15 of the well-known engineering design problems are also determined to evaluate the overall performance of the proposed method. The best results of different classical and new metaheuristic optimization algorithms in dealing with the selected problems were taken from the recent literature for comparison with MGA. Additionally, the statistical values of the MGA algorithm, consisting of the mean, worst, and standard deviation, were calculated and compared to the results of other metaheuristic algorithms. Overall, this work demonstrates that the proposed MGA is able provide very competitive, and even outstanding, results and mostly outperforms other metaheuristics.
\end{abstract}

Keywords: material generation algorithm; constrained problems; metaheuristic algorithm; optimization; engineering design problem

\section{Introduction}

Optimization techniques have been proposed for the optimum design of different problems of everyday life in order to increase the efficiency of systems and human resources. Most of the design problems in nature are complex, with multiple design variables and constraints that classical optimization algorithms, such as gradient-based algorithms, cannot handle. As a solution, numerous artificial intelligence experts have introduced new algorithms with better performance in different fields. Regarding the recent developments in technology, new optimization methods offering higher efficiency, greater accuracy, and increased speed rate are required to deal with difficult optimization problems.

Based on the mentioned concerns about the capabilities of optimization algorithms, a "metaheuristic" approach has been proposed by optimization experts [1] for solving different optimization problems. 'Metaheuristic' refers to specific solution techniques, where higher-level strategies are implemented into the main searching process of the optimization algorithms to provide a powerful searching method with specific capabilities, including the avoidance of entrapment in local optimal solutions. The history of developing different metaheuristic approaches as solutions in different optimization fields can be classified into five different time periods. A brief summary of these historical time periods is presented in Table 1. 
Table 1. Summary of historical time periods for the evolution of metaheuristics [2].

\begin{tabular}{|c|c|c|}
\hline Duration & Period & Achievement \\
\hline Pre-1940 & Pre-Theoretical & Limited applications without formal presentation. \\
\hline 1940-1980 & Early & Introduction of heuristics approaches. \\
\hline 1980-2000 & Method-Centric & $\begin{array}{c}\text { Proposal and improvement of metaheuristics algorithms for different } \\
\text { applications. }\end{array}$ \\
\hline 2000-Present & Framework-Centric & Utilization of metaheuristic frameworks in different fields. \\
\hline Future & Scientific or Future & $\begin{array}{c}\text { Future development and design of metaheuristics as a matter of science } \\
\text { rather than a matter of art. }\end{array}$ \\
\hline
\end{tabular}

With the evolution of numerous metaheuristic algorithms, four different types could be distinguished in terms of their main concepts and inspirations. The first category includes "evolutionary algorithms," such as the Memetic Algorithm (MA) [3], Genetic Algorithm (GA) [4], Genetic Programming (GP) [5], Differential Evolution (DE) [6], Evolution Strategies (ES) [7], and the Biogeography-Based Optimizer (BBO) [8], that have been proposed based on the biological reproduction and evolution. The second category contains swarm intelligence-based optimization algorithms, which are based on the cooperative behavior of self-organized and decentralized artificial or natural systems. Some well-known methods of this category are Particle Swarm Optimization (PSO) [9], Ant Colony Optimization (ACO) [10], Artificial Bee Colony (ABC) [11], Cat Swarm Optimization (CSA) [12], Firefly Algorithm (FA) [13], and Krill Herd (KH) algorithm [14]. The third category consists of algorithms that are motivated by physical laws, such as Simulated Annealing (SA) [15], Harmony Search (HS) [16], Big-Bang Big-Crunch (BBBC) [17], Gravitational Search Algorithm (GSA) [18], Charged System Search (CSS) algorithm [19], Artificial Chemical Reaction Optimization Algorithm (ACROA) [20], Colliding Bodies Optimization (CBO) [21], Chaos Game Optimization (CGO) [22,23], and Atomic Orbital Search (AOS) [24] algorithm. Finally, metaheuristic approaches inspired by the lifestyle of animals or humans are classified in the fourth category, which includes Imperialistic Competitive Algorithm (ICA) [25], Cuckoo Search Algorithm (CSA) [26]. In addition to these metaheuristic algorithms, other difficult challenges have been solved by upgrading, developing, and hybridizing standard algorithms [27-36].

In this paper, a novel metaheuristic algorithm called the Material Generation Algorithm (MGA) is proposed as an alternative approach for solving optimization problems. The main concept of this novel algorithm is based on the principles of chemistry, regarding the production of new materials according to the configurations of chemical compounds and reactions. To evaluate the performance of MGA, we tested it on 15 well-known engineering design problems and 10 constrained mathematical problems in different dimensions (10, 30,50, and 100), which have been benchmarked by the Competitions on Evolutionary Computation (CEC) and presented in detail by Wu et al. [37] at CEC 2017. The utilized references include the results of CEC 2017, Tvrdík and Poláková [38], Polakova [39], and Zamuda [40]. The Friedman Test [41] is also conducted as a well-known statistical test in order to have a fair judgment about the performance of the MGA.

In recent decades there has be a great challenge for the algorithm developers to develop new solution methods which could have better performance than the previous methods in dealing with complex real-world problems. Due to the massive emergence of novel metaheuristic algorithms in the past few decades, this aspect has been addressed by Sorensen [42] as a tsunami of methods which will have advantages and also disadvantages in the soft computing fields in the future. However, this issue can be justified by discovering other aspects of proposing novel algorithms which is based on the source of inspirational concept of a novel algorithm which should be reasonable enough to be justified alongside a well-developed mathematical model as two of the most important principles of metaheuristic algorithms. Regarding the fact that when a novel algorithm is proposed, it is evaluated by some of the benchmark test problems which has been solved 
by multiple methods in order to demonstrate its capability as an independent algorithm among the other methods while this kind of proposing a testing the algorithms is not the only aim of this area. The proposed novel algorithm can be of a great help in the situations that the other alternatives cannot reach to a reasonable response in dealing with a considered problem so there should be other alternatives in order to have a good chance to provide a well-designed plan for the industry and even human-related actions in the everyday life. A brief outline of this work is as follows:

Section 2 discusses the inspirational concept and mathematical model of the MGA optimization algorithm. In Section 3, the problem statements, including the selected mathematical and engineering optimization problems utilized to test the proposed MGA as a novel metaheuristic algorithm, are presented. In Sections 4 and 5, the numerical results of the MGA algorithm and other alternative metaheuristic methods in dealing with the considered mathematical and engineering optimization problems are presented. In Section 6, the key findings of this research work are concluded, future research directions are suggested.

\section{Material Generation Algorithm}

In this section, the inspiration of MGA as a novel metaheuristic algorithm and the mathematical model of this algorithm are presented.

\subsection{Inspiration}

A material is a mixture of multiple substances comprised of the stuffs of the universe with volume and mass. The material generation process concerns the capability of different substances to merge with each other in order to generate new materials with higher functionality and improved energy levels. Elements are the basic building blocks of the materials, which cannot be broken into parts or even changed into other elements. Materials are engineered on an atomic, nano-, micro-, or macro-scale in order to control the specific properties and improve the performance of a material. Uniquely-generated materials are classified based on their general properties and specific characteristics and according to physical and chemical changes that influence a material's behavior.

Material chemistry is one of the most important disciplines in the material research field. Material engineers study the configuration of materials in order to improve the specific characteristics of materials, developing new ones that are more sustainable and also superior to the previous ones. Chemical changes in materials are achieved by reacting and combining various chemicals. In general, the chemical properties are altered by the transferring or sharing of electrons between atoms of different materials, specifically, chemical bonds formed between materials result in such modifications. In this work, three main concepts of material chemistry (compounds, reactions, and stability) were considered to formulate a metaheuristic optimization algorithm.

\subsubsection{Chemical Compound}

Most chemical elements in the universe are created through combinations with other elements. With that being, a few chemical elements exist freely in nature. Compounds are formed by combining multiple chemicals via chemical bonds, or the transferring or sharing of electrons, which result in one of the following:

- Ionic compounds are created when electrons are transferred from the atoms of one element to those of another.

- Covalent compounds form when electrons are shared between atoms of different elements.

In addition, ionic compounds contain multiple ions that are held together by the electrostatic force called ionic bonding. Although these compounds are neutral in nature, they consist of some negatively- and positively-charged ions, called anions and cations, respectively. The evaporation, precipitation, or freezing of the constituent ions are the main factors in the process of producing ionic compounds. When an atom or a small group of atoms starts to lose or gain electrons, an ionic compound forms according to the ionic 
bonding and charged particles. As an example, the formation of sodium chloride, also known as table salt, is depicted in Figure 1. In the process of electron transformation, a sodium (neutral) becomes a sodium cation $\left(\mathrm{Na}^{+}\right)$when it loses one electron. In addition, $\mathrm{Cl}$ becomes a chloride anion $\left(\mathrm{Cl}^{-}\right)$when it gains an electron. Thus, table salt is a solid aggregation of $\mathrm{Na}^{+}$and $\mathrm{Cl}^{-}$ions, which attract each other due to opposite charges.

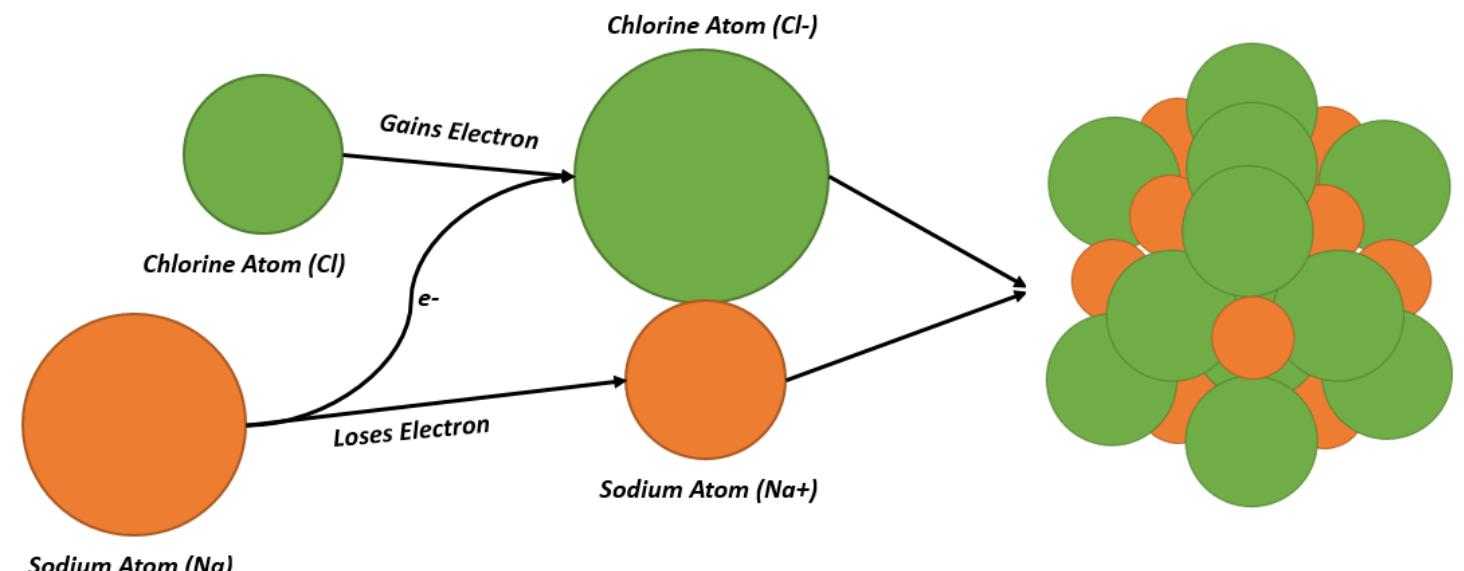

Figure 1. The formation of an ionic compound, $\mathrm{NaCl}$.

Covalent compounds form when an atom of a chemical element shares an electron with another element's atom, which usually occurs between nonmetal elements and results in an electrically neutral atom. Figure 2 displays the formation of a covalent compound that leads to the hydrogen atom. As an example, assuming that two hydrogen atoms begin approaching each other, the nucleus of one atom strongly attracts the electron of the other one. A covalent bond is achieved when a specific distance between the nuclei is reached, and the electrons are equally shared. The net repulsion between nuclei is ignored due to the greater net attraction.

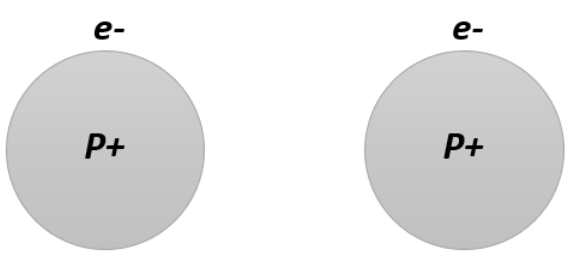

No Interactions

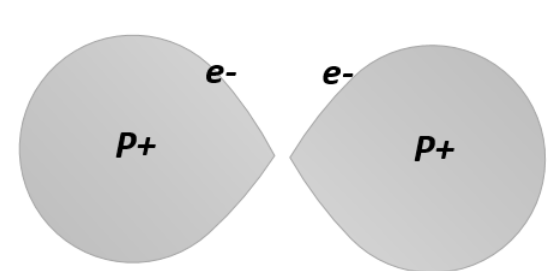

Attractions Begins

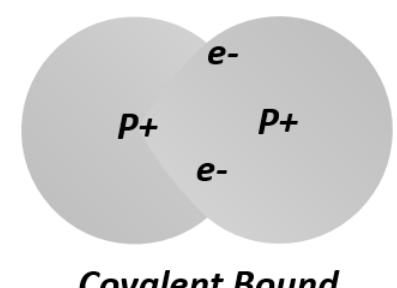

Figure 2. The formation of a covalent compound by means of two hydrogen atoms.

\subsubsection{Chemical Reaction}

Chemical reactions are the process of transforming one material into another while the chemical equations are used to represent chemical reactions, where the resulting products will have different properties than the starting materials (reactants/reagents), and intermediate materials (in some particular cases). 
An example of a chemical reaction is depicted in Figure 3, in which the magnesium wire $(\mathrm{Mg})$ and oxygen gas $\left(\mathrm{O}_{2}\right)$ yield powdery magnesium oxide $(\mathrm{MgO})$. As presented in the left bulb, a fine magnesium filament is surrounded by oxygen before the reaction occurs. As the reaction proceeds, the white colored powdery magnesium oxide coats the bulb's inner surface, which is demonstrated in the right bulb. In this reaction, heat and light are also produced as intermediate materials but are not concerned in this description. The chemical equation of the presented chemical reaction is as follows:

$$
2 \mathrm{Mg}(\mathrm{s})+\mathrm{O}_{2}(\mathrm{~g}) \stackrel{\text { Electricity }}{\rightarrow} 2 \mathrm{MgO}(\mathrm{s})
$$

where s and g stand for solid and gas, respectively.
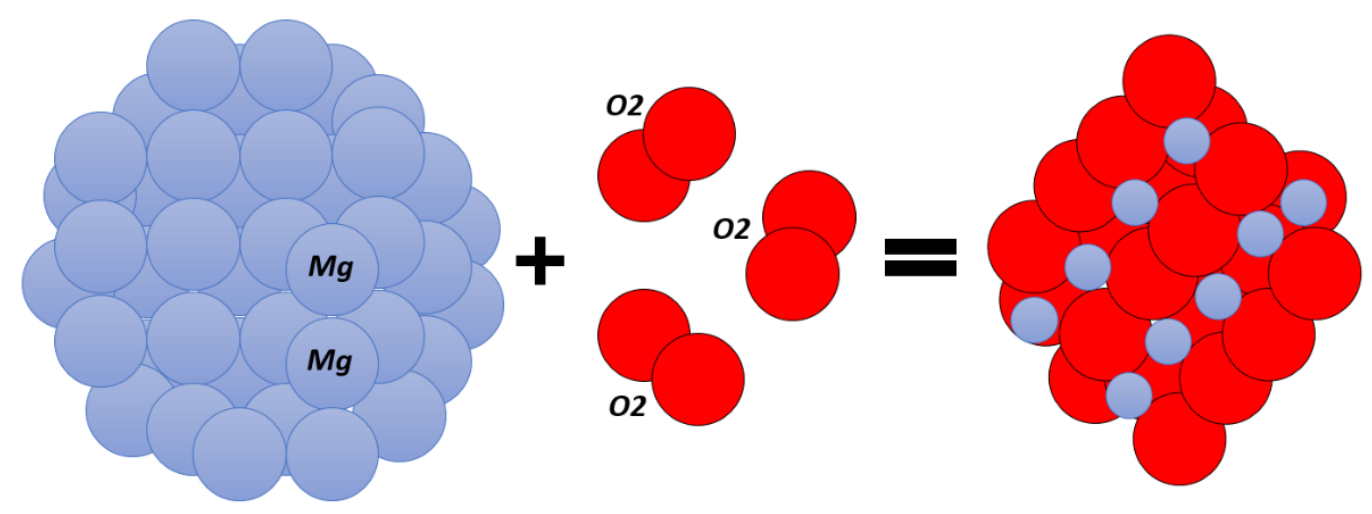

Figure 3. The formation of a chemical reaction.

\subsubsection{Chemical Stability}

Stability is one of the more important properties of materials in real-world applications. When generating new materials with different characteristics, it is important to consider the stability of the chemical compounds and reactions in different situations. In terms of chemical stability, chemicals have the tendency to resist changes, such as decomposition, due to internal factors and external influences such as heat, air, light, and pressure. Chemical stability is the resistance of a material to change in the presence of other chemicals. A stable chemical product refers to one that has not been specifically reactive in the environment and retains its properties over a specific period of time. Comparatively, unstable chemical materials easily decompose, corrode, polymerize, explode, or burn under certain conditions.

When producing new chemical materials, the processes of transferring or sharing electrons within the initial materials will occur in such a way that the end product will be stable and applicable during a specific period of time.

\subsection{Mathematical Model}

In order to conduct an optimum design procedure, an optimization algorithm is developed in this section based on the mentioned principles of material chemistry. The basic concepts of the chemical compounds, reactions, and stability are utilized in order to develop and formulate a well-defined mathematical model for the new algorithm. Considering that many natural evolution algorithms establish a predefined population of solution candidates that are evolved through random alterations and selection, MGA determines a number of materials (Mat) comprised of multiple periodic table elements $(P T E s)$. In this algorithm, a number of materials is considered as the solution candidates $\left(\mathrm{Mat}_{n}\right)$, which are comprised of some elements represented as decision variables $\left(P T E_{i}^{j}\right)$. The mathematical presentation of these two aspects is as follows: 


$$
M a t=\left[\begin{array}{c}
M a t_{1} \\
M_{2} t_{2} \\
\vdots \\
M^{2} t_{i} \\
\vdots \\
M_{n} t_{n}
\end{array}\right]=\left[\begin{array}{cccccc}
P T E_{1}^{1} & P T E_{1}^{2} & \cdots & P T E_{1}^{j} & \cdots & P T E_{1}^{d} \\
P T E_{2}^{1} & P T E_{2}^{2} & \cdots & P T E_{2}^{j} & \cdots & P T E_{2}^{d} \\
\vdots & \vdots & & \vdots & \ddots & \vdots \\
P T E_{i}^{1} & P T E_{i}^{2} & \cdots & P T E_{i}^{j} & \cdots & P T E_{i}^{d} \\
\vdots & \vdots & & \vdots & \ddots & \vdots \\
P T E_{n}^{1} & P T E_{n}^{2} & \cdots & P T E_{n}^{j} & \cdots & P T E_{n}^{d}
\end{array}\right],\left\{\begin{array}{l}
i=1,2, \ldots, n . \\
j=1,2, \ldots, d .
\end{array}\right.
$$

where $d$ is the number of elements (decision variables) in each material (solution candidates); and $n$ is the number of materials considered to be the solution candidates.

In the first stage of the optimization process, $P T E_{i}^{j}$ is determined randomly while the decision variables bounds are defined based on the considered problem. The initial positions of PTEs are determined randomly in the search space as follows:

$$
\operatorname{PTE}_{i}^{j}(0)=P T E_{i, \text { min }}^{j}+\operatorname{Unif}(0,1) \cdot\left(\operatorname{PTE}_{i, \text { max }}^{j}-P T E_{i, \text { min }}^{j}\right),\left\{\begin{array}{l}
i=1,2, \ldots, n . \\
j=1,2, \ldots, d .
\end{array}\right.
$$

where $P T E_{i}^{j}(0)$ determines the initial value of the $j$ th element in the $i$ th material; $P T E_{i, \text { min }}^{j}$ and $P T E_{i, \max }^{j}$ are the minimum allowable and maximum allowable values for the $j$ th decision variable of the $i$ th solution candidate, respectively; and Unif $(0,1)$ is a random number in the interval of $[0,1]$.

\subsubsection{Modeling Chemical Compound}

To mathematically model the chemical compounds, all PTEs are assumed to be in the ground state, which can be externally excited by the magnetic fields, absorption of energy from photons or light and interactions with different colliding bodies or particles regarding ions or other individual electrons. Due to the different stabilities of elements, they have a tendency to lose, gain, or even share electrons with other PTEs, resulting in ionic or covalent compounds. To model the ionic and covalent compounds, $d$ random PTEs are selected using the initial Mat (Equation (1)). For the selected PTEs, the processes of losing, gaining, or sharing electrons are modeled through the probability theory. To fulfill this aim, a continuous probability distribution is utilized for each PTE to configure a chemical compound, which is considered as a new PTE, as follows:

$$
P T E_{\text {new }}^{k}=P T E_{r_{1}}^{r_{2}} \pm e^{-}, k=1,2, \ldots, d .
$$

where $r_{1}$ and $r_{2}$ are uniformly distributed random integers in the intervals of $[1, n]$ and [1, d], respectively; $P T E_{r_{1}}^{r_{2}}$ is a randomly selected PTE from the Mat; $e^{-}$is the probabilistic component for modeling the process of losing, gaining or sharing electrons represented with normal Gaussian distribution in the mathematical model; and $P T E_{\text {new }}^{k}$ is the new material.

The newly-created PTEs are utilized for producing a new material $\left(M a t_{n e w}\right)$, which is then added to the initial material list (Mat) as a new solution candidate:

$$
\text { Mat }_{\text {new }}=\left[P T E_{\text {new }}^{1} P T E_{\text {new }}^{2} \cdots P T E_{\text {new }}^{k} \cdots P T E_{\text {new }}^{d}\right], k=1,2, \ldots, d .
$$

Then, the overall solution candidates are combined and presented as follows: 


$$
M a t=\left[\begin{array}{c}
M^{2} t_{1} \\
M_{2} t_{2} \\
\vdots \\
M a t_{i} \\
\vdots \\
M a t_{n} \\
\text { Mat }_{n e w}
\end{array}\right]=\left[\begin{array}{cccccc}
P T E_{1}^{1} & P T E_{1}^{2} & \cdots & P T E_{1}^{j} & \cdots & P T E_{1}^{d} \\
P T E_{2}^{1} & P T E_{2}^{2} & \cdots & P T E_{2}^{j} & \cdots & P T E_{2}^{d} \\
\vdots & \vdots & & \vdots & \ddots & \vdots \\
P T E_{i}^{1} & P T E_{i}^{2} & \cdots & P T E_{i}^{j} & \cdots & P T E_{i}^{d} \\
\vdots & \vdots & & \vdots & \ddots & \vdots \\
P T E_{n}^{1} & P T E_{n}^{2} & \cdots & P T E_{n}^{j} & \cdots & P T E_{n}^{d} \\
P T E_{\text {new }}^{1} & P T E_{\text {new }}^{2} & \cdots & P T E_{\text {new }}^{k} & \cdots & P T E_{\text {new }}^{d}
\end{array}\right],\left\{\begin{array}{l}
i=1,2, \ldots, n . \\
j=1,2, \ldots, d . \\
k=1,2, \ldots, d .
\end{array}\right.
$$

A schematic presentation of the described process for the configuration of new materials based on the concept of chemical compounds (ionic and covalent) is depicted in Figure 4.

$$
\begin{aligned}
& M a t=\left[\begin{array}{cccccc}
P T E_{1}^{1} & \left(\begin{array}{l}
r_{1}=1 \\
r_{2}=2
\end{array}\right. & & & & \\
P T E_{1}^{2} & \cdots & P T E_{1}^{j} & \cdots & P T E_{1}^{d} \\
P T E_{2}^{1} & P T E_{2}^{2} & \cdots & P T E_{2}^{j} & \cdots & P T E_{2}^{d,} \\
\vdots & \vdots & & \vdots & \ddots & \vdots \\
\hdashline P T E_{i}^{1} & P T E_{i}^{2} & \cdots & P T E_{i}^{j} & \cdots & P T E_{i}^{d} \\
\hdashline & \vdots & & \vdots & \ddots & \vdots \\
P T E_{n}^{1} & P T E_{n}^{2} & \cdots & P T E_{n}^{j !} & \cdots & P T E_{n}^{d}
\end{array}\right],\left\{\begin{array}{l}
r_{1}=2 \\
r_{2}=d
\end{array}\right.
\end{aligned}
$$

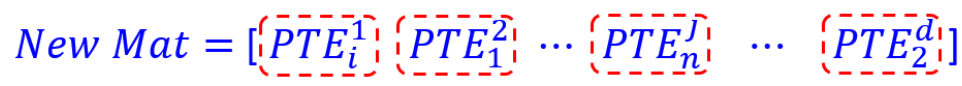

Figure 4. The schematic presentation of the random periodic table elements (PTE) selection and creating new materials.

The probabilistic approach for determining $e^{-}$is modeled through normal Gaussian distribution, which is important in statistics and often used in the natural and social sciences to represent real-valued random variables with unknown distributions. The probability of selecting a new element $\left(P T E_{\text {new }}^{k}\right)$ regarding the randomly selected initial element $\left(P T E_{r_{1}}^{r_{2}}\right)$ is presented as follows:

$$
f\left(\operatorname{PTE}_{\text {neww }}^{k} \mid \mu, \sigma^{2}\right)=\frac{1}{\sqrt{2 \pi \sigma^{2}}} \cdot e^{\frac{-(x-\mu)^{2}}{2 \sigma^{2}}}, k=1,2, \ldots, d .
$$

where $\mu$ is the mean, median or expectation of the distribution correspond to the selected random PTE $\left(P T E_{r_{1}}^{r_{2}}\right) ; \sigma$ is the standard deviation, which is set to unity in this paper; $\sigma^{2}$ is the variance; and $e$ is the natural base or Naperian base of the natural logarithm.

\subsubsection{Modeling Chemical Reaction}

Chemical reactions are sort of production process in which different chemical changes are determined in order to produce different products with modified properties even different from the initial reactants. In order to mathematically model the process of producing new materials by the chemical reaction concept, an integer random number $(l)$ is determined regarding the number of materials of the initial Mat are considered for participating in a chemical reaction. Then, $l$ integer random numbers $(m j)$ are generated to determine the positions of the selected materials in the initial Mat so, the new solutions are linear combinations of the other solutions. For each material, a participation factor $(p)$ is also calculated since different materials would participate in the reactions with different 
amounts. A schematic presentation of the described process is depicted in Figure 5, and the mathematical presentation is as follows:

$$
\operatorname{Mat}_{n e w_{2}}=\frac{\sum_{m=1}^{l}\left(p_{m} \cdot M a t_{m j}\right)}{\sum_{m=1}^{l}\left(p_{m j}\right)}, j=1,2, \ldots, l .
$$

where $M a t_{m}$ is the $m$ th randomly selected material from the initial Mat; $p_{m}$ is the normal Gaussian distribution for the $m$ th material participation factor; and $M a t_{n e w}$ is the new material produced by the chemical reaction concept.

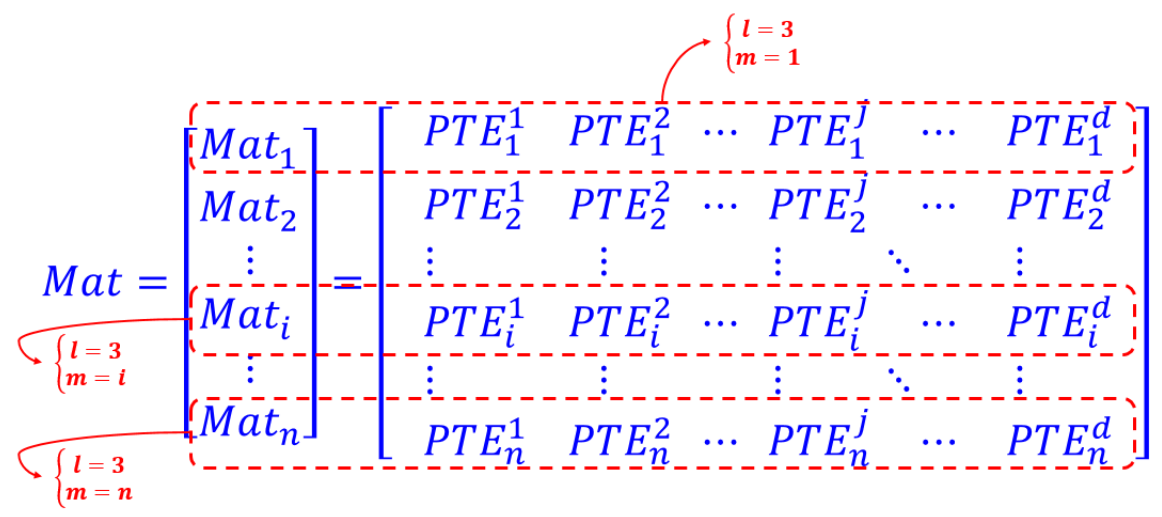

$$
\begin{aligned}
& \text { New Mat }=\left[\frac{\sum_{m=1}^{l}\left(p_{m} \cdot M a t_{m}\right)}{\sum_{m=1}^{l}\left(p_{m}\right)}\right]
\end{aligned}
$$

Figure 5. The schematic view of the random material selection for creating new materials.

\subsubsection{Modeling Chemical Stability}

As previously described, the principle of material stability concerns the tendency of natural systems to seek local and general equilibria at all structural levels. Material stability is mathematically represented by determining the quality of the solutions as Mat. Materials with the highest stability levels alongside the ones with lowest stability levels are equivalent to the best and worst fitness values of all solution candidates in the optimization runs.

Considering the chemical compound and chemical reaction configuration approaches, the overall solution candidates are combined as follows:

$$
M a t=\left[\begin{array}{c}
\mathrm{Mat}_{1} \\
\mathrm{Mat}_{2} \\
\vdots \\
\mathrm{Mat}_{i} \\
\vdots \\
M a t_{n} \\
\mathrm{Mat}_{\text {new1 }} \\
\mathrm{Mat}_{\text {new } 2}
\end{array}\right], i=1,2, \ldots, n
$$

Moreover, the stability levels of the initial material and newly0produced materials should be considered in order to decide whether or not the new materials should be included in the overall material list (Mat) corresponding to the solution candidates. The quality of new solution candidates is then compared to the initial ones, whereby the new materials should be substituted by initial materials with worst fitness values corresponding to worst stability levels.

For boundary violation control, a flag is determined in order to control the violating solution candidates while a maximum number of iteration or objective function evaluation 
can be considered as stopping criteria. The flowchart of the MGA algorithm is presented in Figure 6.

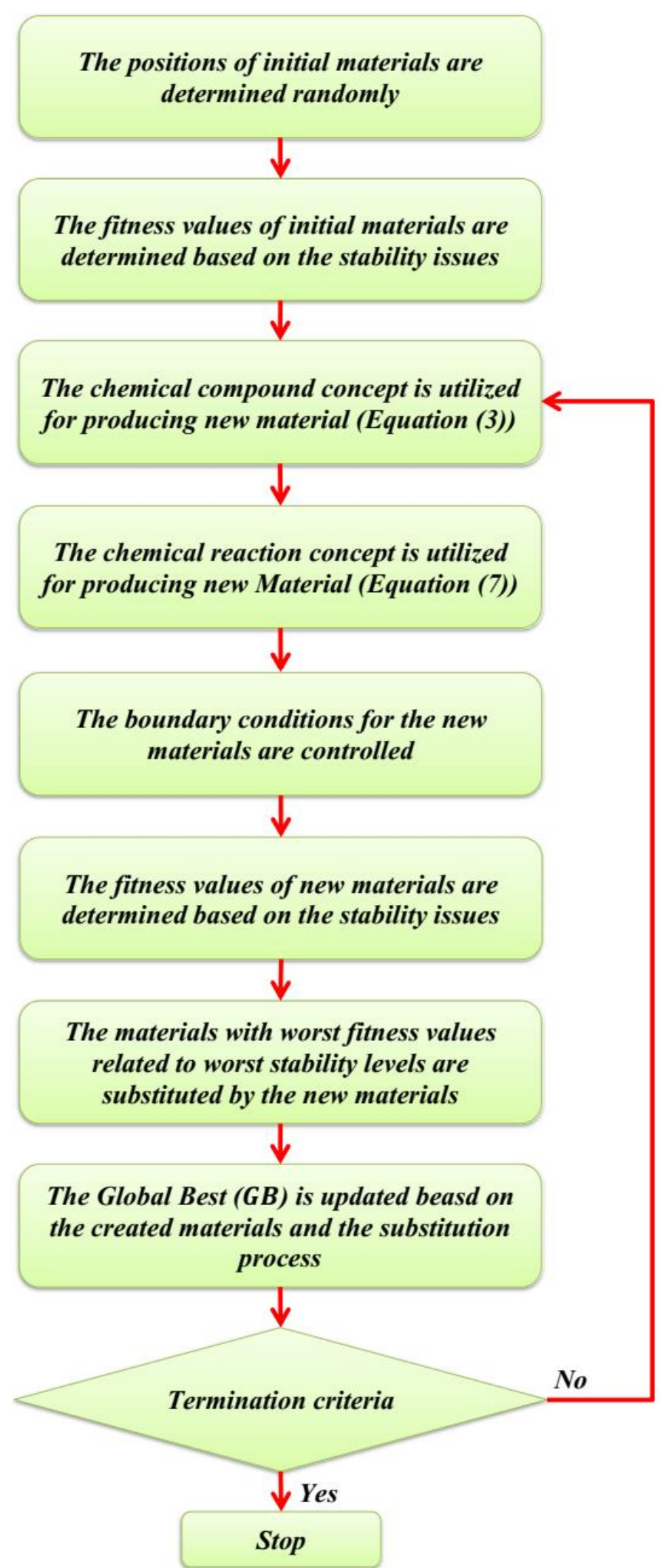

Figure 6. Flowchart of the Material Generation Algorithm (MGA). 


\section{Problem Statement}

In this section, a brief description of the considered design examples is presented. Regarding the fact that these examples are categorized as constrained optimization problems, the general formulations of these kinds of optimization problems are presented as follows:

$$
\begin{gathered}
f(\bar{x}), \quad \bar{x}=x_{1}, x_{2}, \ldots, x_{n} \\
g_{i}(\bar{x}) \leq 0, \quad i=1,2, \ldots, n \\
h_{j}(\bar{x})=0, \quad j=1,2, \ldots, m
\end{gathered}
$$

where $f(\bar{x})$ is considered as the objective function of the optimization problem that can be considered to be maximized or minimized; $g_{i}(\bar{x})$ and $h_{j}(\bar{x})$ are the $i$ th and $j$ th inequality and equality constraint, respectively; $\bar{x}$ is the position vector related to the optimization variables; and $n$ and $m$ are the total number of inequality and equality constraints, respectively.

In most cases, the equality constraints can be transformed into inequality constraints by considering the following:

$$
\left|h_{j}(\bar{x})\right|-\varepsilon \leq 0, j=1,2, \ldots, m
$$

where $\varepsilon$ is a predefined small positive number, which is typically near to zero. In this work, $\varepsilon$ was set to 0.0001 .

\subsection{Mathematically-Constrained Problems}

The mathematical problems of the CEC 2017 benchmark suite are presented in Table 2, while the specific details and mathematical formulations were presented in detail by $\mathrm{Wu}$ et al. [39]. In order to evaluate the results of the proposed MGA, the statistical results of different state-of-the-art metaheuristic algorithms regarding the considered constrained problems were derived of the recent literature [38-40].

Table 2. Brief description of the Competitions on Evolutionary Computation (CEC) 2017 mathematical constrained problems [37].

\begin{tabular}{cccccc}
\hline No. & Type & D & H & G & Bounds \\
\hline$C_{1}$ & Non Separable & $10,30,50$ and 100 & 0 & 1 & $-100 \leq \mathrm{x}_{\mathrm{i}} \leq 100$ \\
$\mathrm{C}_{2}$ & Non Separable & $10,30,50$ and 100 & 0 & 1 & $-100 \leq \mathrm{x}_{\mathrm{i}} \leq 100$ \\
$\mathrm{C}_{3}$ & Non Separable & $10,30,50$ and 100 & 1 & 1 & $-100 \leq \mathrm{x}_{\mathrm{i}} \leq 100$ \\
$\mathrm{C}_{4}$ & Separable & $10,30,50$ and 100 & 0 & 2 & $-10 \leq \mathrm{x}_{\mathrm{i}} \leq 10$ \\
$\mathrm{C}_{5}$ & Non Separable & $10,30,50$ and 100 & 0 & 2 & $-10 \leq \mathrm{x}_{\mathrm{i}} \leq 10$ \\
$\mathrm{C}_{6}$ & Separable & $10,30,50$ and 100 & 6 & 0 & $-20 \leq \mathrm{x}_{\mathrm{i}} \leq 20$ \\
$\mathrm{C}_{7}$ & Separable & $10,30,50$ and 100 & 2 & 0 & $-50 \leq \mathrm{x}_{\mathrm{i}} \leq 50$ \\
$\mathrm{C}_{8}$ & Separable & $10,30,50$ and 100 & 2 & 0 & $-100 \leq \mathrm{x}_{\mathrm{i}} \leq 100$ \\
$\mathrm{C}_{9}$ & Separable & $10,30,50$ and 100 & 2 & 0 & $-10 \leq \mathrm{x}_{\mathrm{i}} \leq 10$ \\
$\mathrm{C}_{10}$ & Separable & $10,30,50$ and 100 & 2 & 0 & $-100 \leq \mathrm{x}_{\mathrm{i}} \leq 100$ \\
\hline
\end{tabular}

D: Dimensions; G: Number of inequality constraints; H: Number of equality constraints.

\subsection{Engineering Design Problems}

The second type of constrained problems included 15 well-known engineering problems, which have been solved by different optimization algorithms. A brief description of these design examples is presented in Table 3, and the specific details of each example are provided in the following subsections. These examples have also been benchmarked by Kumar et al. [43] regarding the CEC 2020 engineering design scheme. 
Table 3. Description of the constrained engineering design problems.

\begin{tabular}{cccccc}
\hline No. & Name & D & G & H & Formulation \\
\hline$F_{1}$ & Speed Reducer & 7 & 11 & 0 & {$[44]$} \\
$\mathrm{F}_{2}$ & Tension/Compression Spring & 3 & 4 & 0 & {$[45]$} \\
$\mathrm{F}_{3}$ & Pressure Vessel & 4 & 4 & 0 & {$[45]$} \\
$\mathrm{F}_{4}$ & Welded Beam & 4 & 7 & 0 & {$[45]$} \\
$\mathrm{F}_{5}$ & Three-Bar Truss & 2 & 3 & 0 & {$[46]$} \\
$\mathrm{F}_{6}$ & Multiple Disk Clutch Brake & 5 & 8 & 0 & {$[47]$} \\
$\mathrm{F}_{7}$ & Planetary Gear Train & 9 & 10 & 1 & {$[48]$} \\
$\mathrm{F}_{8}$ & Step-Cone Pulley & 5 & 8 & 3 & {$[49]$} \\
$\mathrm{F}_{9}$ & Hydrostatic Thrust Bearing & 4 & 7 & 0 & {$[50]$} \\
$\mathrm{F}_{10}$ & Ten-Bar Truss & 10 & 3 & 0 & {$[51]$} \\
$\mathrm{F}_{11}$ & Rolling Element Bearing & 10 & 9 & 0 & {$[52]$} \\
$\mathrm{F}_{12}$ & Gear Train & 4 & 1 & 1 & {$[53]$} \\
$\mathrm{F}_{13}$ & Steel I-Shaped Beam & 4 & 2 & 0 & {$[46]$} \\
$\mathrm{F}_{14}$ & Piston Lever & 4 & 4 & 0 & {$[46]$} \\
$\mathrm{F}_{15}$ & Cantilever Beam & 5 & 1 & 0 & {$[46]$} \\
\hline
\end{tabular}

D: Dimensions; G: Number of inequality constraints; H: Number of equality constraints; Min: Feasible Solutions.

\section{Numerical Results of Mathematical Problems}

The numerical results based on the CEC 2017 benchmark problems by means of the MGA and other alternatives in dealing with the described constrained problems with different dimensions of 10, 30, 50, and 100 are presented in this section. For comparison, a total of 25 optimization runs was performed, including a maximum number of function evaluations $(20,000 \times \mathrm{D})$, where $\mathrm{D}$ is the problem dimension. These results are presented in Tables 4-7 for different dimensions, in which (c) is the number of violated constraints consisting of the number of violations by more than $1,0.01$, and $0.0001 ;(\overline{\mathrm{v}})$ is the mean violation at the median solution; (SR) is the feasibility rate defined as the ratio of feasible runs to total runs; and ( $\overline{\text { vio }}$ ) is the mean constraint violation values of all optimization runs.

Based on the obtained results of MGA in dealing with the mathematical constrained problems of CEC 2017 with a dimension of 10, MGA was superior to the other metaheuristics in most of the cases. Considering the functions with dimensions of 30, MGA outranks two of the alternative metaheuristics while in comparing to the third one, the results of MGA are so competitive. In dealing with functions of 50 and 100 dimensions, the results of MGA are comparable to the others.

Regarding the fact that the considered problems of the CEC 2017 benchmark suite are all the latest problems in the evolutionary computation field with higher levels of complexity and difficulties while there are few approaches that can provide acceptable results in dealing with these problems. In this regard, the reported results by MGA are marginal because there are not any better results for the considered problems in the literature so the MGA calculated the latest reported results which demonstrate the capability of this algorithm in competing with other methods.

In order to have a better perspective on the performance of different metaheuristic algorithms in dealing with the CEC 2017 benchmark problems, the box plots which are derived of the analysis of the variance (ANOVA), which were conducted for the normalized values of the reported bests, means, standard deviations (Std), and worsts for different dimensions of 10, 30, 50, and 100 in Figures 7-10. It can be concluded that the MGA has competitive performance in dealing with these problems. 
Table 4. Statistical results of different approaches for mathematical problems of CEC 2017 with 10 dimensions.

\begin{tabular}{|c|c|c|c|c|c|c|c|c|c|c|c|}
\hline \multirow{2}{*}{ Reference } & \multirow{2}{*}{ Result } & \multicolumn{10}{|c|}{ Function } \\
\hline & & $\mathrm{C}_{1}$ & $\mathrm{C}_{2}$ & $\mathrm{C}_{3}$ & $\mathrm{C}_{4}$ & $\mathrm{C}_{5}$ & $\mathrm{C}_{6}$ & $\mathrm{C}_{7}$ & $\mathrm{C}_{8}$ & $\mathrm{C}_{9}$ & $\mathrm{C}_{10}$ \\
\hline \multirow{9}{*}{ Zamuda [38] } & Best & 0 & 0 & 62700 & 13.573 & 0 & 332.30 & -178.02 & -0.00135 & -0.00498 & -0.00051 \\
\hline & Median & 0 & 0 & $2.260 \times 10^{5}$ & 13.573 & 0 & 1750.6 & -26.778 & -0.00135 & -0.00498 & -0.00051 \\
\hline & c & $0,0,0$ & $0,0,0$ & $0,0,0$ & $0,0,0$ & $0,0,0$ & $0,4,2$ & $0,0,0$ & $0,0,0$ & $0,0,0$ & $0,0,0$ \\
\hline & $\overline{\mathrm{V}}$ & 0 & 0 & 0 & 0 & 0 & $3.83 \times 10^{-2}$ & 0 & 0 & 0 & 0 \\
\hline & Mean & 0 & 0 & $3.259 \times 10^{5}$ & 14.418 & 0 & 808.36 & -34 & 0 & 0 & 0 \\
\hline & Worst & 0 & 0 & $1.089 \times 10^{6}$ & 15.919 & 0 & 1819.7 & -7 & 0 & 0 & 0 \\
\hline & Std & 0 & 0 & $2.575 \times 10^{5}$ & 1.1495 & 0 & 545.03 & 57 & 0 & 0 & 0 \\
\hline & SR & 100 & 100 & 100 & 100 & 100 & 0 & 80 & 100 & 100 & 100 \\
\hline & $\overline{\text { vio }}$ & 0 & 0 & 0 & 0 & 0 & $3.766 \times 10^{-2}$ & $3.189 \times 10^{-5}$ & 0 & 0 & 0 \\
\hline \multirow{8}{*}{$\begin{array}{c}\text { Polakova } \\
\text { [39] }\end{array}$} & Best & 0 & 0 & 3533.77 & 13.5728 & 0 & 348.977 & -101.211 & -0.00135 & -0.00498 & -0.00051 \\
\hline & Median & 0 & 0 & $21,144.4$ & 13.5853 & 0 & 1368.85 & 12.7815 & -0.00135 & -0.00498 & -0.00051 \\
\hline & C & $0,0,0$ & $0,0,0$ & $0,0,0$ & $0,0,0$ & $0,0,0$ & $0,4,2$ & $0,0,0$ & $0,0,0$ & $0,0,0$ & $0,0,0$ \\
\hline & $\overline{\mathrm{v}}$ & 0 & 0 & 0 & 0 & 0 & 0.029702 & 0 & 0 & 0 & 0 \\
\hline & Mean & 0 & 0 & $31,548.2$ & 13.6147 & 0 & 648.899 & 3.74362 & -0.00135 & -0.00497 & -0.00051 \\
\hline & Worst & 0 & 0 & 118,005 & 13.8018 & 0 & 1260.3 & 105.62 & -0.00135 & -0.00485 & -0.00051 \\
\hline & Std & 0 & 0 & $37,019.6$ & 0.061549 & 0 & 283.706 & 69.5716 & $2.21 \times 10^{-19}$ & $2.44 \times 10^{-5}$ & $1.11 \times 10^{-19}$ \\
\hline & $\overline{\text { vio }}$ & 0 & 0 & $6.67 \times 10^{-6}$ & 0 & 0 & 0.032309 & $2.11 \times 10^{-5}$ & 0 & 0 & 0 \\
\hline \multirow{9}{*}{$\begin{array}{c}\text { Tvrdík and } \\
\text { Poláková } \\
\text { [40] }\end{array}$} & Best & 0 & 0 & 6341.810292 & 15.919244 & 0 & 103.288465 & -148.219878 & -0.001348 & -0.004975 & -0.000510 \\
\hline & Median & 0 & 0 & $40,103.1993$ & 35.818324 & 0 & 307.643490 & -65.209283 & -0.001348 & -0.004975 & -0.000510 \\
\hline & c & $0,0,0$ & $0,0,0$ & $0,0,1$ & $0,0,0$ & $0,0,0$ & $0,0,5$ & $0,0,2$ & $0,0,2$ & $0,0,1$ & $0,0,1$ \\
\hline & $\overline{\mathrm{V}}$ & 0 & 0 & 0.000103 & 0 & 0 & 0 & 0 & 0 & 0 & 0 \\
\hline & Mean & 0 & 0 & 110,008 & 38.738 & 0.956779 & 549.617 & -48.7352 & -0.001348 & 0.125471 & -0.00051 \\
\hline & Worst & 0 & 0 & $548,034.199888$ & 55.717399 & 3.986579 & 2058.812018 & 102.366112 & -0.001348 & 3.256178 & -0.000510 \\
\hline & Std & 0 & 0 & $1.5587 \times 10^{5}$ & $8.948 \times 10^{5}$ & $1.737 \times 10$ & $4.866 \times 10^{2}$ & $6.826 \times 10^{1}$ & $6.639 \times 10^{-19}$ & $6.522 \times 10^{-1}$ & 0.0000 \\
\hline & SR & 100 & 100 & 44 & 100 & 100 & 96 & 68 & 100 & 100 & 100 \\
\hline & $\overline{\text { vio }}$ & 0 & 0 & 0.00063352 & 0 & 0 & 0.0053656 & 0.00309144 & $1.456 \times 10^{-5}$ & $4 \times 10^{-6}$ & $3.96 \times 10^{-6}$ \\
\hline \multirow{4}{*}{$\begin{array}{c}\text { Present } \\
\text { Study (MGA) }\end{array}$} & Best & 0 & 0 & 5731.729 & 15.91932 & 0.048494 & 177.1936 & -204.799 & -0.00103 & -0.00497 & -0.00048 \\
\hline & Median & 0 & 0 & 9655.116 & 18.9044315 & 1.554645 & 189.7318 & -99.5936 & 0.000667 & -0.00497 & -0.00034 \\
\hline & C & $0,0,0$ & $0,0,0$ & $0,0,0$ & $0,0,0$ & $0,0,0$ & $0,4,2$ & $0,0,0$ & $0,0,0$ & $0,0,0$ & $0,0,0$ \\
\hline & $\overline{\mathrm{v}}$ & 0 & 0 & 0 & 0 & 0 & 0.070346 & 0 & 0 & 0 & 0 \\
\hline
\end{tabular}


Table 4. Cont.

\begin{tabular}{|c|c|c|c|c|c|c|c|c|c|c|c|}
\hline \multirow{2}{*}{ Reference } & \multirow{2}{*}{ Result } & \multicolumn{10}{|c|}{ Function } \\
\hline & & $\mathrm{C}_{1}$ & $\mathrm{C}_{2}$ & $\mathrm{C}_{3}$ & $\mathrm{C}_{4}$ & $\mathrm{C}_{5}$ & $\mathrm{C}_{6}$ & $\mathrm{C}_{7}$ & $\mathrm{C}_{8}$ & $\mathrm{C}_{9}$ & $\mathrm{C}_{10}$ \\
\hline & Mean & 0 & 0 & $22,532.64$ & 18.57982 & 1.867275 & 245.6745 & -86.6422 & 0.001115 & 0.04604 & -0.0003 \\
\hline & Worst & 0 & 0 & $116,693.6$ & 27.8597111 & 4.016201 & 1231.20125 & 8.612641 & 0.008756 & 0.57474405 & $8.06 \times 10^{-5}$ \\
\hline & Std & 0 & 0 & $35,636.84$ & 4.235729 & 1.393692 & 308.6091 & 68.43618 & 0.002994 & 0.152843 & 0.000167 \\
\hline & SR & 100 & 100 & 100 & 100 & 100 & 11 & 100 & 89 & 100 & 100 \\
\hline & $\overline{\text { vio }}$ & 0 & 0 & 0 & 0 & 0 & 0.059761 & 0 & $1.11 \times 10^{-5}$ & 0 & 0 \\
\hline
\end{tabular}

Table 5. Statistical results of different approaches for mathematical problems of CEC 2017 with 30 dimensions.

\begin{tabular}{|c|c|c|c|c|c|c|c|c|c|c|c|}
\hline \multirow{2}{*}{ Reference } & \multirow{2}{*}{ Result } & \multicolumn{10}{|c|}{ Function } \\
\hline & & $\mathrm{C}_{1}$ & $\mathrm{C}_{2}$ & $\mathrm{C}_{3}$ & $\mathrm{C}_{4}$ & $\mathrm{C}_{5}$ & $\mathrm{C}_{6}$ & $C_{7}$ & $\mathrm{C}_{8}$ & $\mathrm{C}_{9}$ & $\mathrm{C}_{10}$ \\
\hline \multirow{9}{*}{$\begin{array}{c}\text { Zamuda } \\
\text { [38] }\end{array}$} & Best & 0 & 0 & $2.76 \times 10^{6}$ & 13.573 & 0 & 4095.8 & -234.05 & $-2.82 \times 10^{-4}$ & -0.00267 & -0.000103 \\
\hline & Median & 0 & 0 & $6.58 \times 10^{6}$ & 13.573 & 0 & 4374.9 & -80.772 & $-2.70 \times 10^{-4}$ & -0.00267 & $-9.91 \times 10^{-5}$ \\
\hline & c & $0,0,0$ & $0,0,0$ & $0,0,0$ & $0,0,0$ & $0,0,0$ & $0,4,2$ & $0,0,0$ & $0,0,0$ & $0,0,0$ & $0,0,0$ \\
\hline & $\overline{\mathrm{V}}$ & 0 & 0 & 0 & 0 & 0 & $2.55 \times 10^{-2}$ & 0 & 0 & 0 & 0 \\
\hline & Mean & 0 & 0 & $6.70 \times 10^{6}$ & 13.854 & 0 & 5526.4 & -81.088 & $-2.63 \times 10^{-4}$ & $-2.67 \times 10^{-3}$ & $-9.78 \times 10^{-5}$ \\
\hline & Worst & 0 & 0 & $1.17 \times 10^{7}$ & 15.919 & 0 & 5018.0 & -36.510 & $-2.12 \times 10^{-4}$ & $-2.67 \times 10^{-3}$ & $-8.96 \times 10^{-5}$ \\
\hline & Std & 0 & 0 & $2.25 \times 10^{6}$ & 0.7782 & 0 & 759.06 & 90.929 & $2.04 \times 10^{-5}$ & $0.00 \times 10^{0}$ & $3.69 \times 10^{-6}$ \\
\hline & SR & 100 & 100 & 100 & 100 & 100 & 0 & 96 & 100 & 100 & 100 \\
\hline & $\overline{\text { vio }}$ & 0 & 0 & 0 & 0 & 0 & $2.57 \times 10^{-2}$ & $4.06 \times 10^{-6}$ & 0 & 0 & 0 \\
\hline \multirow{8}{*}{$\begin{array}{c}\text { Polakova } \\
\text { [39] }\end{array}$} & Best & 0 & 0 & $39,059.8$ & 13.5728 & 0 & 3121.78 & -245.715 & -0.00028 & -0.00267 & -0.0001 \\
\hline & Median & 0 & 0 & 20,5874 & 13.5728 & 0 & 5802.76 & -134.373 & -0.00028 & -0.00267 & -0.0001 \\
\hline & c & $0,0,0$ & $0,0,0$ & $0,0,0$ & $0,0,0$ & $0,0,0$ & $0,4,2$ & $0,0,0$ & $0,0,0$ & $0,0,0$ & $0,0,0$ \\
\hline & $\overline{\mathrm{v}}$ & 0 & 0 & 0 & 0 & 0 & 0.012659 & 0 & 0 & 0 & 0 \\
\hline & Mean & $3.87 \times 10^{-30}$ & $5.26 \times 10^{-30}$ & 355,118 & 13.5728 & 0 & 4071.08 & -109.428 & -0.00028 & -0.00267 & -0.0001 \\
\hline & Worst & $2.08 \times 10^{-29}$ & $3.34 \times 10^{-29}$ & $2.18 \times 10^{6}$ & 13.5728 & 0 & 2405.82 & 81.6284 & -0.00028 & -0.00267 & -0.0001 \\
\hline & Std & $6.10 \times 10^{-30}$ & $8.39 \times 10^{-30}$ & 446,751 & $5.44 \times 10^{-15}$ & 0 & 981.519 & 88.7374 & 0 & $1.33 \times 10^{-18}$ & 0 \\
\hline & vio & 0 & 0 & 0 & 0 & 0 & 0.015016 & $8.20 \times 10^{-6}$ & 0 & 0 & 0 \\
\hline
\end{tabular}


Table 5. Cont.

\begin{tabular}{|c|c|c|c|c|c|c|c|c|c|c|c|}
\hline \multirow{2}{*}{ Reference } & \multirow{2}{*}{ Result } & \multicolumn{10}{|c|}{ Function } \\
\hline & & $\mathrm{C}_{1}$ & $\mathrm{C}_{2}$ & $\mathrm{C}_{3}$ & $\mathrm{C}_{4}$ & $\mathrm{C}_{5}$ & $\mathrm{C}_{6}$ & $\mathrm{C}_{7}$ & $\mathrm{C}_{8}$ & $\mathrm{C}_{9}$ & $\mathrm{C}_{10}$ \\
\hline \multirow{9}{*}{$\begin{array}{c}\text { Tvrdík } \\
\text { and } \\
\text { Poláková } \\
{[40]}\end{array}$} & Best & 0 & 0 & $217,854.405028$ & 64.671883 & 0 & 1976.35821 & -330.786337 & -0.000284 & -0.002666 & -0.000103 \\
\hline & Median & 0 & 0 & $736,404.82$ & 113.424634 & 0 & 3827.58828 & -32.589365 & -0.000284 & -0.002666 & -0.000103 \\
\hline & c & $0,0,0$ & $0,0,0$ & $0,0,1$ & $0,0,0$ & $0,0,0$ & $0,0,4$ & $0,0,2$ & $0,0,2$ & $0,0,1$ & $0,0,1$ \\
\hline & $\overline{\mathrm{V}}$ & 0 & 0 & 0.001441 & 0 & 0 & 0 & 0.000067 & 0 & 0 & 0 \\
\hline & Mean & 0 & 0 & $1.299 \times 10^{6}$ & 115.734 & 0.797325 & 3745.32 & -24.1162 & -0.000284 & 0.0233628 & -0.000103 \\
\hline & Worst & 0 & 0 & $5,082,420.837959$ & 159.192594 & 3.986624 & 5065.298248 & 185.582813 & -0.000284 & 0.648053 & -0.000103 \\
\hline & Std & 0 & 0 & $1.195 \times 10^{6}$ & $2.201 \times 10^{1}$ & $1.627 \times 10$ & $8.431 \times 10^{2}$ & $1.154 \times 10^{2}$ & $1.659 \times 10^{-19}$ & $1.301 \times 10^{-1}$ & $4.149 \times 10^{-20}$ \\
\hline & SR & 100 & 100 & 32 & 100 & 100 & 100 & 52 & 100 & 96 & 100 \\
\hline & $\overline{\text { vio }}$ & 0 & 0 & 0.0242756 & 0 & 0 & $1.164 \times 10^{-5}$ & 0.0035614 & 0 & $1.0709 \times 10^{6}$ & $6.6 \times 10^{-6}$ \\
\hline \multirow{8}{*}{$\begin{array}{l}\text { Present } \\
\text { Study } \\
\text { (MGA) }\end{array}$} & Best & 0 & 0 & $101,125.9$ & 72.90983 & 0 & 1369.466 & -214.361 & 1.311075 & 0.000266 & 0.342705 \\
\hline & Median & 0 & 0 & $3,769,626.34$ & 106.7165 & 0 & 1582.655 & -212.331 & 2.173907 & 0.574744 & 0.627412 \\
\hline & c & $0,0,0$ & $0,0,0$ & $0,0,0$ & $0,0,0$ & $0,0,0$ & $0,1,4$ & $0,0,0$ & $2,0,0$ & $0,0,0$ & $2,0,0$ \\
\hline & $\overline{\mathrm{v}}$ & 0 & 0 & 0 & 0 & 0 & 0.002259 & 0 & 6.590862 & 0 & 2.124071 \\
\hline & Mean & 0 & 0 & $497,341.7$ & 103.0124 & 0 & 1639.729 & -229.997 & 2.013486 & 0.903313 & 0.587699 \\
\hline & Worst & 0 & 0 & $1,083,246$ & 196.971781 & 0 & 2375.443 & -52.3606 & 3.901132 & 4.706474 & 0.887197 \\
\hline & Std & 0 & 0 & $417,284.9$ & 22.09312 & 0 & 459.9857 & 106.7847 & 0.979232 & 1.476608 & 0.206912 \\
\hline & $\overline{\text { vio }}$ & 0 & 0 & 0 & 0 & 0 & 0.010403 & 0 & 3.814903 & 0 & 2.547508 \\
\hline
\end{tabular}

Table 6. Statistical results of different approaches for mathematical problems of CEC 2017 with 50 dimensions.

\begin{tabular}{|c|c|c|c|c|c|c|c|c|c|c|c|}
\hline \multirow{2}{*}{ Reference } & \multirow{2}{*}{ Result } & \multicolumn{10}{|c|}{ Function } \\
\hline & & $\mathrm{C}_{1}$ & $\mathrm{C}_{2}$ & $\mathrm{C}_{3}$ & $\mathrm{C}_{4}$ & $\mathrm{C}_{5}$ & $\mathrm{C}_{6}$ & $\mathrm{C}_{7}$ & $\mathrm{C}_{8}$ & $\mathrm{C}_{9}$ & $\mathrm{C}_{10}$ \\
\hline \multirow{7}{*}{$\begin{array}{c}\text { Zamuda } \\
\text { [38] }\end{array}$} & Best & 0 & 0 & $7.80 \times 10^{6}$ & 13.573 & 0 & 8775 & -347.6 & $1.40 \times 10^{-4}$ & $3.25 \times 10^{-5}$ & -347.6 \\
\hline & Median & 0 & 0 & $2.65 \times 10^{7}$ & 13.573 & 0 & 10,224 & -134.7 & $2.87 \times 10^{-4}$ & $8.66 \times 10^{-5}$ & -134.7 \\
\hline & c & $0,0,0$ & $0,0,0$ & $0,0,0$ & $0,0,0$ & $0,0,0$ & $0,1,5$ & $0,0,0$ & $0,0,0$ & $0,0,0$ & $0,0,0$ \\
\hline & $\overline{\mathrm{V}}$ & 0 & 0 & 0 & 0 & 0 & $1.38 \times 10^{-2}$ & 0 & 0 & 0 & 0 \\
\hline & Mean & $1.49 \times 10^{-8}$ & 0 & $2.65 \times 10^{7}$ & 13.988 & 0 & 8601 & -154.0 & $2.86 \times 10^{-4}$ & $9.12 \times 10^{-5}$ & -154.0 \\
\hline & Worst & $1.00 \times 10^{-7}$ & $5.94 \times 10^{-8}$ & $4.25 \times 10^{7}$ & 16.914 & 0 & 9202 & 39.3 & $4.85 \times 10^{-4}$ & $2.28 \times 10^{-4}$ & 39.3 \\
\hline & Std & $1.95 \times 10^{-8}$ & $1.17 \times 10^{-8}$ & $8.66 \times 10^{6}$ & 0.9868 & 0 & 1217 & 106.3 & $8.44 \times 10^{-5}$ & $3.91 \times 10^{-5}$ & 106.3 \\
\hline
\end{tabular}


Table 6. Cont

\begin{tabular}{|c|c|c|c|c|c|c|c|c|c|c|c|}
\hline \multirow{2}{*}{ Reference } & \multirow{2}{*}{ Result } & \multicolumn{10}{|c|}{ Function } \\
\hline & & $\mathrm{C}_{1}$ & $\mathrm{C}_{2}$ & $\mathrm{C}_{3}$ & $\mathrm{C}_{4}$ & $\mathrm{C}_{5}$ & $\mathrm{C}_{6}$ & $\mathrm{C}_{7}$ & $\mathrm{C}_{8}$ & $\mathrm{C}_{9}$ & $\mathrm{C}_{10}$ \\
\hline & SR & 100 & 100 & 100 & 100 & 100 & 0 & 100 & 100 & 100 & 100 \\
\hline & $\overline{\text { vio }}$ & 0 & 0 & 0 & 0 & 0 & $1.52 \times 10^{-2}$ & 0 & 0 & 0 & 0 \\
\hline \multirow{8}{*}{$\begin{array}{c}\text { Polakova } \\
\text { [39] }\end{array}$} & Best & $8.68 \times 10^{-30}$ & $2.50 \times 10^{-29}$ & 286,730 & 13.5728 & 0 & 6708.83 & -0.00013 & -0.00204 & $-4.83 \times 10^{-5}$ & -0.00013 \\
\hline & Median & $7.73 \times 10^{-29}$ & $1.02 \times 10^{-28}$ & 633,683 & 13.5728 & $\begin{array}{l}1.30 \times \\
10^{-28}\end{array}$ & 8636.68 & -0.00013 & -0.00204 & $-4.83 \times 10^{-5}$ & -0.00013 \\
\hline & c & $0,0,0$ & $0,0,0$ & $0,0,0$ & $0,0,0$ & $0,0,0$ & $0,2,4$ & $0,0,0$ & $0,0,0$ & $0,0,0$ & $0,0,0$ \\
\hline & Mean & $7.79 \times 10^{-29}$ & $9.79 \times 10^{-29}$ & 894521 & 13.5728 & $\begin{array}{l}1.68 \times \\
10^{-28}\end{array}$ & 7514.8 & -0.00013 & -0.00204 & $-4.83 \times 10^{-5}$ & -0.00013 \\
\hline & Worst & $1.42 \times 10^{-28}$ & $1.78 \times 10^{-28}$ & $3.87 \times 10^{6}$ & 13.5728 & $\begin{array}{l}6.40 \times \\
10^{-28}\end{array}$ & 6637.22 & -0.00013 & -0.00204 & $-4.83 \times 10^{-5}$ & -0.00013 \\
\hline & Std & $3.08 \times 10^{-29}$ & $4.60 \times 10^{-29}$ & 740490 & $5.44 \times 10^{-15}$ & $\begin{array}{l}1.59 \times \\
10^{-28}\end{array}$ & 1417.76 & $2.77 \times 10^{-20}$ & $1.33 \times 10^{-18}$ & 0 & $2.77 \times 10^{-20}$ \\
\hline & SR & 100 & 100 & 100 & 100 & 100 & 0 & 100 & 100 & 100 & 100 \\
\hline & $\overline{\text { vio }}$ & 0 & 0 & 0 & 0 & 0 & 0.011693 & 0 & 0 & 0 & 0 \\
\hline \multirow{8}{*}{$\begin{array}{c}\text { Tvrdík and } \\
\text { Poláková } \\
\text { [40] }\end{array}$} & Best & 0 & 0 & $460,407.836$ & 145.263065 & 0 & 3486.644298 & -340.22487 & 0.000601 & -0.002037 & -0.00004 \\
\hline & c & $0,0,0$ & $0,0,0$ & $0,0,1$ & $0,0,0$ & $0,0,0$ & $0,0,4$ & $0,0,2$ & $0,0,0$ & $0,0,1$ & $0,0,0$ \\
\hline & $\overline{\mathrm{v}}$ & 0 & 0 & 0.000050 & 0 & 0 & 0 & 0.000075 & 0 & 0 & 0 \\
\hline & Mean & 0 & 0 & $6.6413 \times 10^{6}$ & 187.37 & 0.31893 & 6364.72 & -68.1059 & 0.0009928 & 0.0810008 & $-4.284 \times 10^{-5}$ \\
\hline & Worst & 0 & 0 & $27,234,258.492770$ & 244.758532 & 3.986624 & 9005.415965 & 163.958553 & 0.001558 & 1.138593 & -0.00001 \\
\hline & Std & 0 & 0 & $5.9790 \times 10^{6}$ & $2.5905 \times 10^{1}$ & $\begin{array}{c}1.1038 \times \\
10^{0}\end{array}$ & $1.6322 \times 10^{3}$ & $1.3458 \times 10^{2}$ & $2.4328 \times 10^{-4}$ & $2.3626 \times 10^{-1}$ & $6.101 \times 10^{-6}$ \\
\hline & SR & 100 & 100 & 48 & 100 & 100 & 100 & 56 & 100 & 84 & 100 \\
\hline & $\overline{\text { vio }}$ & 0 & 0 & 0.0694317 & 0 & 0 & $1.02 \times 10^{-5}$ & 0.00180008 & $3.48 \times 10^{-6}$ & $1.4708 \times 10^{7}$ & 0 \\
\hline \multirow{9}{*}{$\begin{array}{c}\text { Present } \\
\text { Study } \\
\text { (MGA) }\end{array}$} & Best & $7.73 \times 10^{-6}$ & $3.40 \times 10^{-7}$ & $67,5040.5$ & 214.0131 & 183.3693 & 2104.094 & -287.24 & 6.111175 & 16.76229 & 11.98384 \\
\hline & Median & $6.16 \times 10^{-6}$ & $2.87 \times 10^{-5}$ & 1686140 & 231.3667 & 264.3097 & 2453.639 & -121.342 & 10.1111743 & 19.39083 & 19.33752 \\
\hline & c & $0,0,0$ & $0,0,0$ & $0,0,0$ & $0,0,0$ & $0,0,0$ & $0,0,0$ & $0,0,0$ & $2,0,0$ & $1,0,0$ & $2,0,0$ \\
\hline & $\overline{\mathrm{V}}$ & 0 & 0 & 0 & 0 & 0 & 0 & 0 & 77.75325 & 1.398507 & 2716.256 \\
\hline & Mean & $2.29 \times 10^{-5}$ & $9.39 \times 10^{-5}$ & $3,733,884$ & 236.5529 & 295.9803 & 2601.021 & -100.093 & 7.705596 & 18.78359 & 23.86641 \\
\hline & Worst & $8.70 \times 10^{-5}$ & 0.000571 & $65,265,448.5$ & 309.4055 & 429.773726 & 4601.16137 & 152.382091 & 10.65774 & 19.76821 & 54.4833554 \\
\hline & Std & $3.41 \times 10^{-5}$ & 0.000167 & $4,614,030$ & 32.99758 & 125.1962 & 591.8259 & 105.2148 & 1.261924 & 0.963696 & 11.58393 \\
\hline & SR & 100 & 100 & 100 & 100 & 100 & 76 & 100 & 0 & 0 & 0 \\
\hline & $\overline{\text { vio }}$ & 0 & 0 & 0 & 0 & 0 & 0.057435 & 0 & 75.19797 & 1.255203 & 3025.091 \\
\hline
\end{tabular}


Table 7. Statistical results of different approaches for mathematical problems of CEC 2017 with 100 dimensions.

\begin{tabular}{|c|c|c|c|c|c|c|c|c|c|c|c|}
\hline \multirow{2}{*}{ Reference } & \multirow{2}{*}{ Result } & \multicolumn{10}{|c|}{ Function } \\
\hline & & $\mathrm{C}_{1}$ & $\mathrm{C}_{2}$ & $\mathrm{C}_{3}$ & $\mathrm{C}_{4}$ & $\mathrm{C}_{5}$ & $\mathrm{C}_{6}$ & $\mathrm{C}_{7}$ & $\mathrm{C}_{8}$ & $\mathrm{C}_{9}$ & $C_{10}$ \\
\hline \multirow{9}{*}{$\begin{array}{c}\text { Zamuda } \\
\text { [38] }\end{array}$} & Best & 2.434 & 1.072 & $9.39 \times 10^{7}$ & 13.573 & 0 & 15,440 & -530.12 & $1.22 \times 10^{-3}$ & $3.51 \times 10^{-4}$ & -530.12 \\
\hline & Median & 6.211 & 2.318 & $2.27 \times 10^{8}$ & 13.573 & 0 & 15,595 & -324.99 & $1.44 \times 10^{-3}$ & $4.13 \times 10^{-4}$ & -324.99 \\
\hline & $\mathrm{c}$ & $0,0,0$ & $0,0,0$ & $0,0,0$ & $0,0,0$ & $0,0,0$ & $0,4,2$ & $0,0,0$ & $0,0,0$ & $0,0,0$ & $0,0,0$ \\
\hline & $\overline{\mathrm{V}}$ & 0 & 0 & 0 & 0 & 0 & $1.18 \times 10^{-2}$ & 0 & 0 & 0 & 0 \\
\hline & Mean & 7 & 3 & $2.25 \times 10^{8}$ & 14.028 & 0 & 15,533 & -335.49 & $1.48 \times 10^{-3}$ & $4.25 \times 10^{-4}$ & -335.49 \\
\hline & Worst & 16.527 & 6.765 & $4.21 \times 10^{8}$ & 16.914 & 0 & 14,830 & -110.18 & $1.78 \times 10^{-3}$ & $5.53 \times 10^{-4}$ & -110.18 \\
\hline & Std & 3.190 & 1.397 & $9.21 \times 10^{7}$ & 1.083 & 0 & 1604 & 122.40 & $1.77 \times 10^{-4}$ & $4.92 \times 10^{-5}$ & 122.40 \\
\hline & SR & 100 & 100 & 100 & 100 & 100 & 0 & 100 & 100 & 100 & 100 \\
\hline & $\overline{\text { vio }}$ & 0 & 0 & 0 & 0 & 0 & $1.19 \times 10^{-2}$ & 0 & 0 & 0 & 0 \\
\hline \multirow{9}{*}{$\begin{array}{c}\text { Polakova } \\
\text { [39] }\end{array}$} & Best & $1.30 \times 10^{-26}$ & $1.31 \times 10^{-26}$ & $1.34 \times 10^{6}$ & 13.5728 & $\begin{array}{c}4.34 \times \\
10^{-7}\end{array}$ & $17,164.3$ & $-4.83 \times 10^{-5}$ & -0.00143 & $-1.72 \times 10^{-5}$ & $-4.83 \times 10^{-5}$ \\
\hline & Median & $4.50 \times 10^{-26}$ & $4.59 \times 10^{-26}$ & $2.47 \times 10^{6}$ & 13.5728 & $\begin{array}{c}4.90 \times \\
10^{-6}\end{array}$ & $15,803.2$ & $-4.82 \times 10^{-5}$ & -0.00143 & $-1.72 \times 10^{-5}$ & $-4.82 \times 10^{-5}$ \\
\hline & c & $0,0,0$ & $0,0,0$ & $0,0,0$ & $0,0,0$ & $0,0,0$ & $0,1,5$ & $0,0,0$ & $0,0,0$ & $0,0,0$ & $0,0,0$ \\
\hline & $\overline{\mathrm{v}}$ & 0 & 0 & 0 & 0 & 0 & 0.009677 & 0 & 0 & 0 & 0 \\
\hline & Mean & $1.03 \times 10^{-25}$ & $8.47 \times 10^{-26}$ & $2.73 \times 10^{6}$ & 13.7132 & $\begin{array}{c}3.28 \times \\
10^{-5}\end{array}$ & $15,562.2$ & $-4.81 \times 10^{-5}$ & -0.00143 & $-1.72 \times 10^{-5}$ & $-4.81 \times 10^{-5}$ \\
\hline & Worst & $6.51 \times 10^{-25}$ & $6.38 \times 10^{-25}$ & $4.79 \times 10^{6}$ & 15.5748 & 0.000416 & $16,718.9$ & $-4.77 \times 10^{-5}$ & -0.00143 & $-1.71 \times 10^{-5}$ & $-4.77 \times 10^{-5}$ \\
\hline & Std & $1.68 \times 10^{-25}$ & $1.25 \times 10^{-25}$ & 965,593 & 0.462703 & $\begin{array}{c}9.25 \times \\
10^{-5}\end{array}$ & 1595.41 & $1.33 \times 10^{-7}$ & $2.21 \times 10^{-19}$ & $1.29 \times 10^{-8}$ & $1.33 \times 10^{-7}$ \\
\hline & SR & 100 & 100 & 100 & 100 & 100 & 0 & 100 & 100 & 100 & 100 \\
\hline & $\overline{\text { vio }}$ & 0 & 0 & 0 & 0 & 0 & 0.009805 & 0 & 0 & 0 & 0 \\
\hline \multirow{9}{*}{$\begin{array}{c}\text { Tvrdík and } \\
\text { Poláková } \\
\text { [40] }\end{array}$} & Best & 0.080255 & 0.072938 & $1,684,503.31$ & 329.329439 & 0 & $10,950.2096$ & -481.32898 & 0.013288 & 0 & 0.000365 \\
\hline & Median & 0.432564 & 0.184568 & $9,938,948.89$ & 408.925707 & 0.011586 & $15,506.5581$ & -278.65043 & 0.027209 & 0.000217 & 0.000501 \\
\hline & c & $0,0,0$ & $0,0,0$ & $0,0,1$ & $0,0,0$ & $0,0,0$ & $0,0,2$ & $0,0,2$ & $0,0,2$ & $0,0,0$ & $0,0,0$ \\
\hline & $\overline{\mathrm{v}}$ & 0 & 0 & 0.002547 & 0 & 0 & 0 & 0.000198 & 0.000832 & 0 & 0 \\
\hline & Mean & 0.977746 & 0.366104 & $1.51413 \times 10^{7}$ & 413.582 & 0.818836 & $15,222.9$ & -193.458 & 0.0415975 & 0.522499 & 0.00051308 \\
\hline & Worst & 11.315168 & 3.620979 & $60,598,481.7$ & 469.617973 & 4.066555 & $18,535.3302$ & 376.526002 & 0.087460 & 5.348516 & 0.000684 \\
\hline & Std & $2.1781 \times 10$ & $\begin{array}{c}6.9971 \times \\
10^{-1}\end{array}$ & $1.3449 \times 10^{7}$ & $3.6721 \times 10^{1}$ & $\begin{array}{c}1.512 \times \\
10\end{array}$ & $1.7824 \times 10^{3}$ & $2.0127 \times 10^{2}$ & $2.4668 \times 10^{-2}$ & $1.1223 \times 10$ & $7.3482 \times 10^{-5}$ \\
\hline & SR & 100 & 100 & 16 & 100 & 100 & 100 & 40 & 0 & 96 & 100 \\
\hline & vio & 0 & 0 & 0.0242065 & 0 & 0 & $5.6 \times 10^{-6}$ & 0.00436664 & 0.0012406 & $1.564 \times 10^{-5}$ & $1.268 \times 10^{-5}$ \\
\hline
\end{tabular}


Table 7. Cont

\begin{tabular}{|c|c|c|c|c|c|c|c|c|c|c|c|}
\hline \multirow{2}{*}{ Reference } & \multirow{2}{*}{ Result } & \multicolumn{10}{|c|}{ Function } \\
\hline & & $\mathrm{C}_{1}$ & $\mathrm{C}_{2}$ & $\mathrm{C}_{3}$ & $\mathrm{C}_{4}$ & $\mathrm{C}_{5}$ & $\mathrm{C}_{6}$ & $\mathrm{C}_{7}$ & $\mathrm{C}_{8}$ & $\mathrm{C}_{9}$ & $\mathrm{C}_{10}$ \\
\hline \multirow{9}{*}{$\begin{array}{c}\text { Present } \\
\text { Study } \\
\text { (MGA) }\end{array}$} & Best & 79.17725 & 82.46377 & $1,939,226$ & 1035.546 & $149,057.2$ & 4524.706 & -24.7894 & 11.02866 & 16.5913 & 47.71097 \\
\hline & Median & 226.9738 & 216.2455 & $5,229,008$ & 1115.146 & $162,884.7$ & 4982.677 & 68.55985 & 11.59449 & 18.9102539 & 53.43286 \\
\hline & $c$ & $0,0,0$ & $0,0,0$ & $0,0,0$ & $0,0,0$ & $0,0,0$ & $0,0,4$ & $2,0,0$ & $2,0,0$ & $1,0,0$ & $2,0,0$ \\
\hline & $\overline{\mathrm{V}}$ & 0 & 0 & 0 & 0 & 0 & 0.002105 & 1162.713 & 1530.176 & 698.7951 & 102,643 \\
\hline & Mean & 242.9825 & 256.1113 & $7,766,430$ & 1107.661 & $167,178.1$ & 5646.481 & 88.40124 & 11.62023 & 18.23359 & 53.97361 \\
\hline & Worst & 400.539192 & 445.345609 & $21,948,580$ & 1186.64915 & 221,456 & 7418.342 & 402.6898 & 13.20224 & 19.2152074 & 60.58393 \\
\hline & Std & 125.7168 & 167.4498 & $6,395,089$ & 61.20722 & $26,483.47$ & 1233.678 & 177.8638 & 1.155593 & 0.934306 & 4.846915 \\
\hline & SR & 100 & 100 & 100 & 100 & 100 & 0 & 0 & 0 & 0 & 0 \\
\hline & $\overline{\text { vio }}$ & 0 & 0 & 0 & 0 & 0 & 0.305535 & 1485.207 & 1221.619 & 773.7698 & 105,498 \\
\hline
\end{tabular}



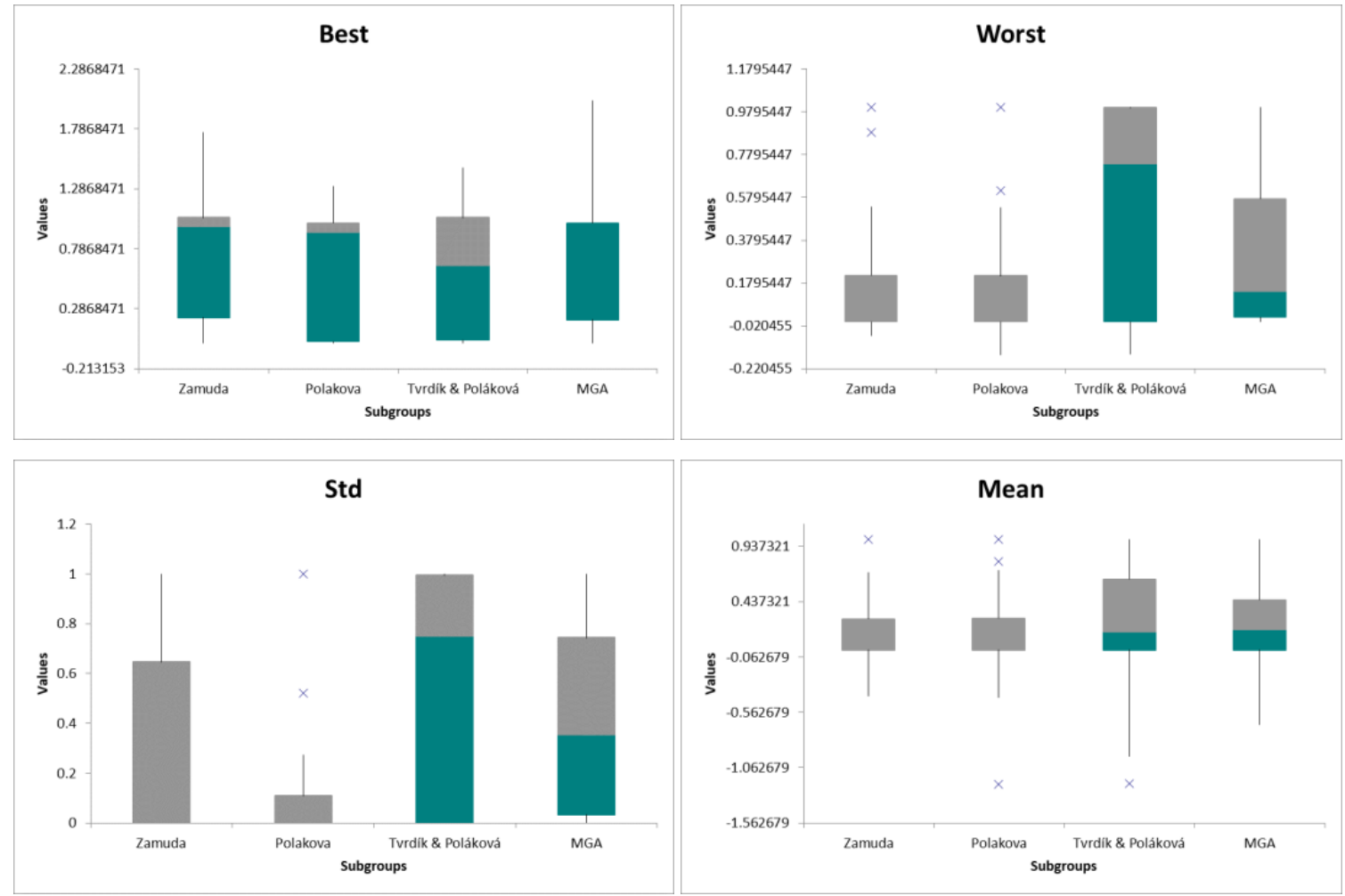

Figure 7. Box plots of the Analysis of Variance (ANOVA) for the 10-dimensional problems.
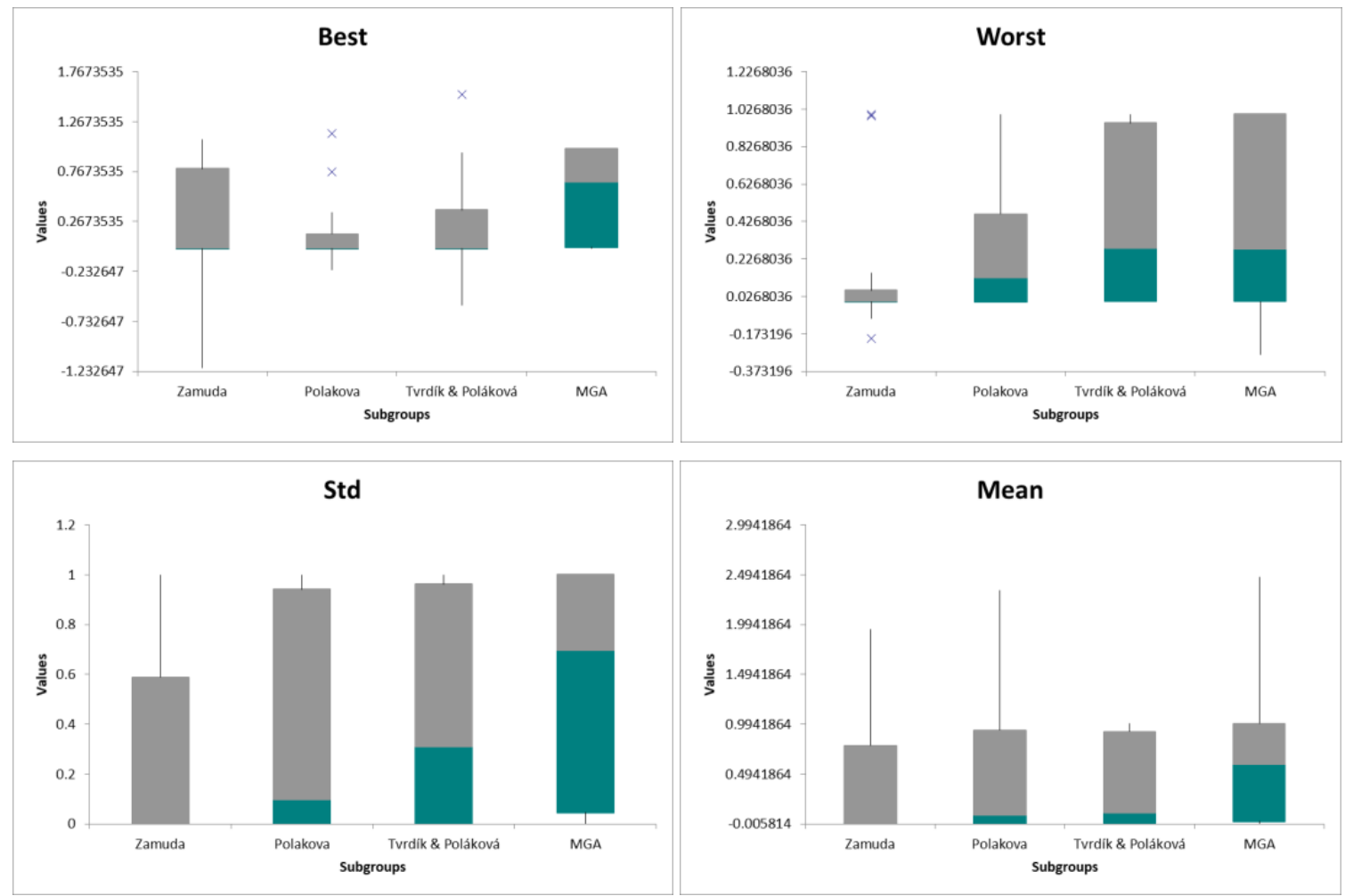

Figure 8. Box plots of the ANOVA for the 30-dimensional problems. 

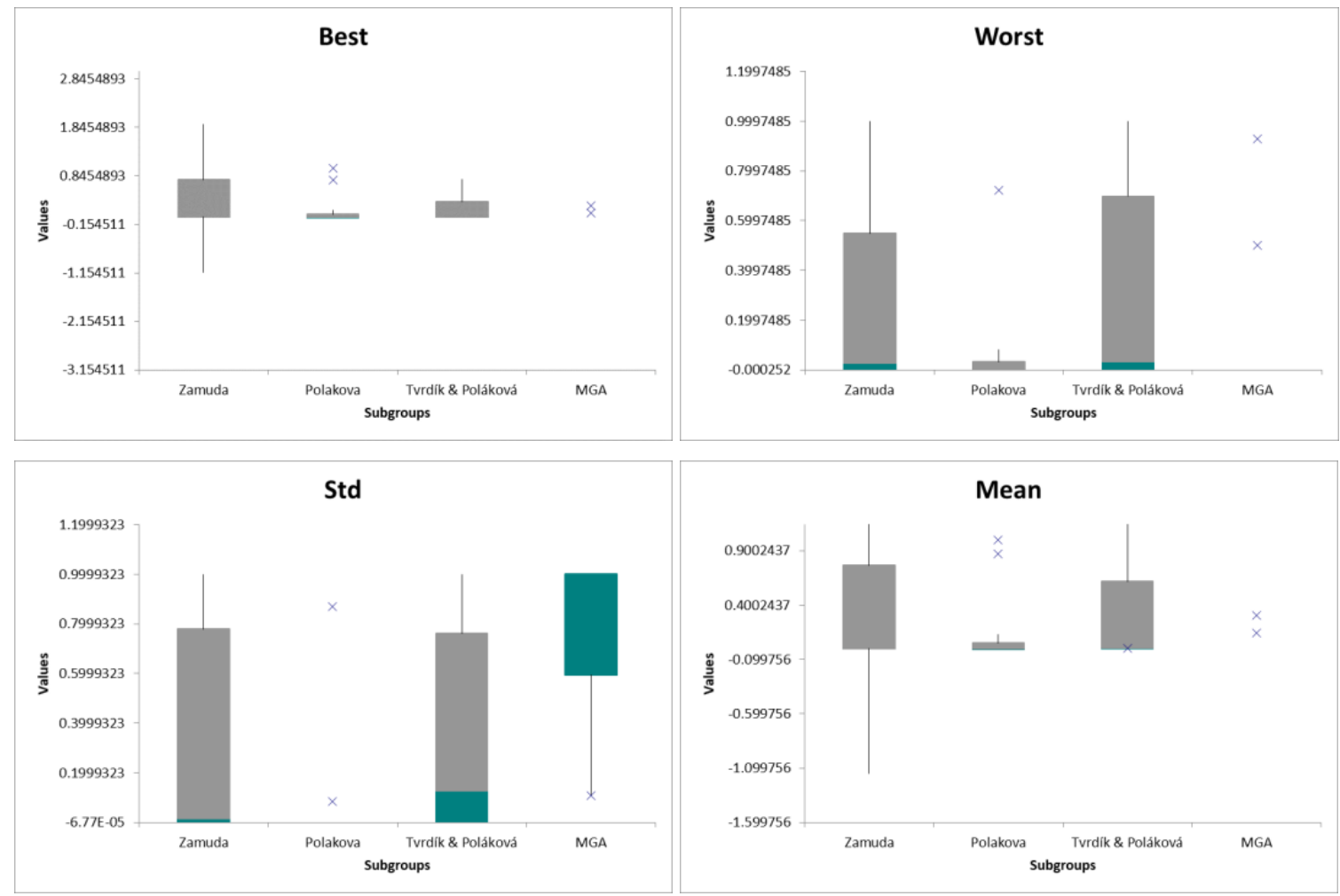

Figure 9. Box plots of the ANOVA for the 50-dimensional problems.
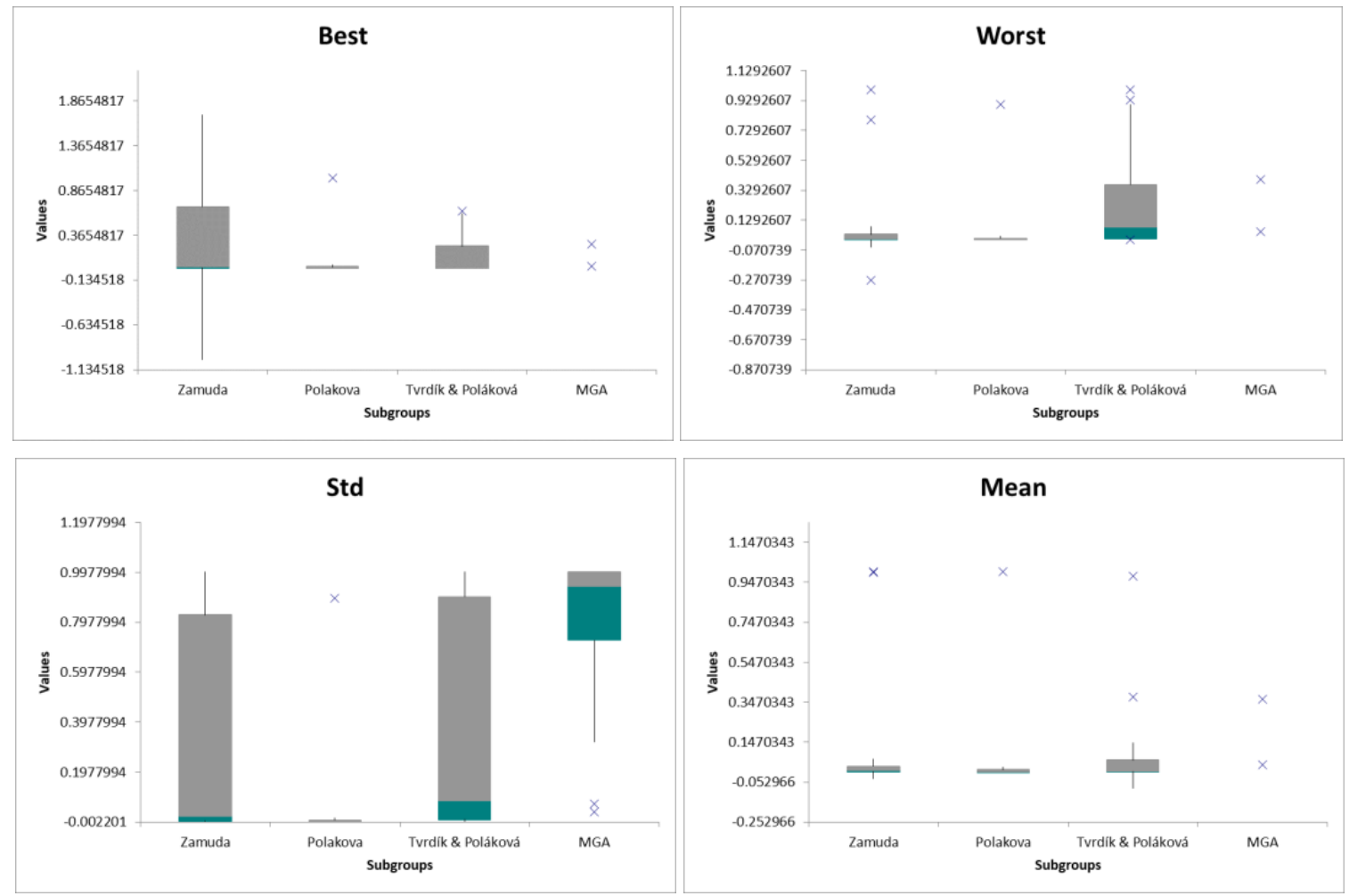

Figure 10. Box plots of the ANOVA for the 100-dimensional problems.

Based on the provided results for the MGA and other state-of-the-art approaches in the evolutionary computation field, the AGA is capable of competing with these excellent algorithms while in some cases even MGA outperforms the others. this performance in 
dealing with CEC 2017 lead to the fact that MGA's mathematical model is well-established model in which the global and local search are conducted with no need to any parameters to be tuned. In other words, this algorithm does not need any internal parameters to be defined prior to the optimization process which makes this algorithm a best choice in dealing with complex problems in which there are not any information about the complexity level of the problem. Additionally, the MGA generates only two new solution candidates in each iteration which makes the algorithm to require less computational efforts for optimization purposes. Hence, these aspects can be of great importance when the MGA is compared to the other metaheuristic algorithms in the evolutionary computation field. In other words, MGA is a parameter free optimization approach with less computational cost, which makes this algorithm different form the other approaches, while the inspirational concept of this algorithm is also unique.

\section{Numerical Results of Engineering Problems}

The numerical results of MGA considering the previously-described engineering design problems are presented in this section. In this regard, the results of other metaheuristics in dealing with these design examples were taken from the literature in order to make fair judgments.

The comparative results of the speed reducer design engineering problem, including the obtained design (decision) variables related to the best optimum configuration determined by different methods, are presented in Table A1. In addition, the statistical results, such as the best, mean, and worst fitness values alongside the standard deviation, are presented in Table 8 . The results of different metaheuristics show that the best results of MGA are better than the best results of the other approaches in dealing with this design example. The MGA is also capable of providing better statistical results, including mean and standard deviation. The Friedman statistical test results are also presented in Table A2 for comparative purposes.

Table 8. Statistical results of different approaches for the speed reducer design problem.

\begin{tabular}{ccccc}
\hline Approaches & Best & Mean & Worst & Std-Dev \\
\hline Montes et al. [54] & 3025.005 & 3088.7778 & 3078.5918 & NA \\
Akhtar et al. [55] & 3008.08 & 3012.1200 & 3028.2800 & NA \\
Gandomi et al. [46] & 3000.9810 & 3007.1997 & 3.0090 & 4.9634 \\
Zhang et al. [56] & 2994.471066 & 2994.471066 & 2994.471066 & $3.58 \times 10^{-12}$ \\
Present Study (MGA) & 2994.438869 & 2994.47065 & 2996.558237 & $4.72 \times 10^{-16}$ \\
\hline
\end{tabular}

Considering the spring design problem, the best and statistical results of different metaheuristics, including the obtained design variables related to the best optimum design, are presented in Tables 9 and A3, respectively. It should be mentioned that MGA is capable of obtaining very competitive results for this constrained engineering design problem. It also should be mentioned that MGA yields better statistical results in terms of the mean, worst fitness values alongside the standard deviation than the results of other metaheuristics. The Friedman statistical test results are also presented in Table A4 for comparative purposes.

Tables 10 and A5 present the final and statistical results obtained by the different methods for the pressure vessel engineering design problem, respectively. From these tables, the best result of the MGA method is better than the results of the other approaches. By comparing the statistical results, it is obvious that MGA has better performance in statistical analysis, especially the mean, and worst fitness values alongside the standard deviation. The Friedman statistical test results are also presented in Table A6 for comparative purposes. 
Table 9. Statistical results of different approaches for the tension or compression spring design problem.

\begin{tabular}{ccccc}
\hline Approaches & Best & Mean & Worst & Std-Dev \\
\hline Coello [57] & 0.01270478 & 0.01276920 & 0.01282208 & $3.9390 \times 10^{-5}$ \\
Ray and Liew [58] & 0.0126692 & 0.0129227 & 0.0167172 & $5.1985 \times 10^{-5}$ \\
Han et al. [59] & 0.01266534 & 0.01268592 & 0.01272968 & $2.1672 \times 10^{-5}$ \\
Gandomi et al. [45] & 0.01266522 & 0.01350052 & 0.0168954 & 0.001420272 \\
Present Study (MGA) & 0.01266523 & 0.01266558 & 0.01266723 & $5.65 \times 10^{-7}$ \\
\hline
\end{tabular}

Table 10. Statistical results of different approaches for the pressure vessel design problem.

\begin{tabular}{ccccc}
\hline Approaches & Best & \multicolumn{1}{c}{ Mean } & Worst & Std-Dev \\
\hline He and Wang [60] & 6061.0777 & 6147.1332 & 6363.8041 & 86.4500 \\
Coelho [61] & 6059.7208 & 6440.3786 & 7544.4925 & 448.4711 \\
Mezura-Montes and & 6059.7456 & 6850.004948 & 7332.879883 & 426 \\
Coello [62] & & & \\
Coello and Montes [63] & 6059.9463 & 6177.2532668 & 6469.32201 & 130.9 \\
Present Study (MGA) & 6059.714350 & 6059.694923 & 6273.765974 & 0.028912058 \\
\hline
\end{tabular}

The results of the welded beam design problem in Tables 11 and A7 show that MGA is capable of converging to better results than the other approaches. Although the maximum difference between the best results of MGA and the other approaches is only about $4 \%$, MGA is capable of providing better statistical results, including the mean, worst fitness values alongside standard deviation. The Friedman statistical test results are also presented in Table A8 for comparative purposes.

Table 11. Statistical results of different approaches for the welded beam design problem.

\begin{tabular}{ccccc}
\hline Approaches & Best & Mean & Worst & Std-Dev \\
\hline Huang et al. [64] & 1.733461 & 1.768158 & 1.824105 & 0.022194 \\
Eskandar et al. [65] & 1.724856 & 1.726427 & 1.744697 & $4.29 \times 10^{-3}$ \\
Guedria [66] & 1.724852 & 1.724853 & 1.724862 & $2.02 \times 10^{-6}$ \\
Han et al. [59] & 1.6956397 & 1.7160908 & 1.7530472 & $1.83 \times 10^{-2}$ \\
Present Study (MGA) & 1.672966512 & 1.678791422 & 1.687172363 & $4.4147 \times 10^{-3}$ \\
\hline
\end{tabular}

In Table A9, the final design of different methods and MGA for the three-bar truss design problem, including the obtained design variables, are presented. Table 12 displays the statistical results. Considering the results reported by previous researchers, it is clear that MGA yields very competitive results for this engineering design problem. MGA determined the best optimum value that has been reported thus far, according to the literature, for the considered design example. It also should be noted that the statistical results, including the mean and standard deviation, for the MGA are much better than the results of other approaches. The Friedman statistical test results are also presented in Table A10 for comparative purposes.

The results of the multiple disk clutch brake design problem solved by MGA and other approaches are summarized in Table A11 [47,50,65,68]. The statistical results are presented in Table 13. Accordingly, MGA is capable of calculating very impressive results compared to the other metaheuristics. The maximum and minimum differences between the results of MGA and other metaheuristics are about $49 \%$ and $24 \%$, which demonstrates the capability of this algorithm in dealing with multiple disk clutch brake design problem. In addition, the statistical results, including the mean and worst fitness values, demonstrate that MGA can yield extremely better results than the other approaches. The Friedman statistical test results are also presented in Table A12 for comparative purposes. 
Table 12. Statistical results of different approaches for the three-bar truss design problem.

\begin{tabular}{ccccc}
\hline Approaches & Best & Mean & Worst & Std-Dev \\
\hline Gandomi et al. [46] & 263.97156 & 264.0669 & NA & 0.00009 \\
Ray and Liew [58] & 263.8958466 & 263.9033 & 263.9033 & $1.26 \times 10^{-2}$ \\
Zhang et al. [56] & 263.8958434 & 263.8958436 & 263.8958498 & $9.72 \times 10^{-7}$ \\
Grag [67] & 263.8958433 & 263.8958437 & 263.8958459 & $5.34 \times 10^{-7}$ \\
Present Study (MGA) & 263.8958433 & 263.8958436 & 263.8959632 & $2.05 \times 10^{-14}$ \\
\hline
\end{tabular}

Table 13. Statistical results of different approaches for the multiple disk clutch brake design problem.

\begin{tabular}{ccccc}
\hline Approaches & Best & Mean & Worst & Std-Dev \\
\hline Eskandar et al. [65] & 0.313656 & 0.313656 & 0.313656 & $1.69 \times 10^{-16}$ \\
Rao et al. [50] & 0.313657 & 0.3271662 & 0.392071 & 0.67 \\
Ferreira et al. [47] & 0.313656 & 0.313656 & 0.313656 & $1.13 \times 10^{-16}$ \\
Present Study (MGA) & 0.235242467 & 0.235244323 & 0.235252239 & $2.42 \times 10^{-6}$ \\
\hline
\end{tabular}

The final results of different metaheuristics in dealing with the planetary gear train design problem, one of the most important and well-established constrained optimization problems, are presented in Tables 14 and A13. By comparing the best results of MGA with other approaches, it can be concluded that MGA can yield outstanding results. Although MGA is also capable of providing better statistical results for the mean and worst fitness values alongside standard deviation results cannot be compared since they have yet to be reported in the literature. The Friedman statistical test results are also presented in Table A14 for comparative purposes.

Table 14. Statistical results of different approaches for the planetary gear train design problem.

\begin{tabular}{ccccc}
\hline Approaches & Best & Mean & Worst & Std-Dev \\
\hline Rao and Savsani [69] (PSO) & 0.53 & 0.5361934 & NA & NA \\
Rao and Savsani [69] (ABC) & 0.525769 & 0.5272922 & NA & NA \\
Zhang et al. [70] & 0.525589 & 0.525589 & NA & NA \\
Savsani and Savsani [48] & 0.525588 & 0.53063 & NA & NA \\
Present Study (MGA) & 0.52325 & 0.5300526 & 0.5370588 & 0.0082564 \\
\hline
\end{tabular}

For the step-cone pulley engineering design problem, the final results of different metaheuristics are presented in Table A15, and the statistical results are provided in Table 15. By comparing the best results, it can be concluded that MGA can yield very impressive results for this constrained engineering problem. The maximum difference between the mean results of MGA and other approaches is about 31\%. The Friedman statistical test results are also presented in Table A16 for comparative purposes.

Table 15. Statistical results of different approaches for the step-cone pulley design problem.

\begin{tabular}{ccccc}
\hline Approaches & Best & Mean & Worst & Std-Dev \\
\hline TLBO [50] & 16.63451 & 24.0113577 & 74.022951 & 0.34 \\
WOA [44] & 16.6345213 & 20.93829477 & 24.8488259 & 3.3498 \\
WCA [44] & 16.63450849 & 17.53037682 & 18.83302997 & 0.9229 \\
MBA [44] & 16.6345078 & 16.702535 & 18.3237145 & 0.2627 \\
Present Study (MGA) & 16.18595608 & 16.35528922 & 16.98647762 & 0.14824361 \\
\hline TLBO: Teaching-Learning-Based Optimization. & & &
\end{tabular}

In Table 16, the comparative results of different metaheuristics in dealing with the hydrostatic thrust bearing design problem, including the obtained design and its related 
best optimum configuration, are presented. Table 17 displays the statistical results. It can be concluded that MGA is capable of converging to better results than the other approaches. The maximum difference between the best results of MGA is about $29 \%$, where MGA yielded better statistical results for the mean, worst fitness values alongside the standard deviation than the other approaches. The Friedman statistical test results are also presented in Table 18 for comparative purposes.

Table 16. Comparison of the best solutions for the hydrostatic thrust bearing design problem.

\begin{tabular}{cccccc}
\hline & Siddall [71] & Deb and Goyal [72] & Coello [73] & Rao et al. [50] & Present Study (MGA) \\
\hline Best & 2288.2268 & 2161.4215 & 1950.2860 & 1625.44276 & 1623.980938 \\
$\mathrm{R}$ & 7.155 & 6.778 & 6.271 & 5.9557805026 & 5.963241516 \\
$\mathrm{R}_{0}$ & 6.689 & 6.234 & 12.901 & 5.3890130519 & 5.395907989 \\
$\mu$ & $8.321 \times 10^{-6}$ & $6.096 \times 10^{-6}$ & $5.605 \times 10^{-6}$ & 0.0000053586 & $5.38 \times 10^{-6}$ \\
$\mathrm{Q}$ & 9.168 & 3.809 & 2.938 & 2.2696559728 & 2.282242505 \\
$\mathrm{~g}_{1}(\mathrm{x})$ & $-11,086.7430$ & -8329.7681 & -2126.86734 & -0.0001374735 & -144.9586796 \\
$\mathrm{~g}_{2}(\mathrm{x})$ & -402.4493 & -177.3527 & -68.0396 & -0.0000010103 & -1.194802021 \\
$\mathrm{~g}_{3}(\mathrm{x})$ & -35.057196 & -10.684543 & -3.705191 & -0.0000000210 & -0.372450027 \\
$\mathrm{~g}_{4}(\mathrm{x})$ & -0.001542 & -0.000652 & -0.000559 & -0.0003243625 & -0.00032915 \\
$\mathrm{~g}_{5}(\mathrm{x})$ & -0.466000 & -0.544000 & -0.666000 & -0.5667674507 & -0.567333527 \\
$\mathrm{~g}_{6}(\mathrm{x})$ & -0.000144 & -0.000717 & -0.000805 & -0.0009963614 & -0.000996355 \\
$\mathrm{~g}_{7}(\mathrm{x})$ & -563.644401 & -83.618221 & -849.718683 & -0.0000090762 & -4.144258876 \\
\hline
\end{tabular}

Table 17. Statistical results of different approaches for the hydrostatic thrust bearing design problem.

\begin{tabular}{ccccc}
\hline Approaches & Best & Mean & Worst & Std-Dev \\
\hline Şahin et al. [74] & 1625.46467 & 1627.744198 & 1650.698747 & 3.815546973 \\
Rao and Waghmare [75] & 1625.44271 & 1796.89367 & 2104.3776 & 0.21 \\
Rao et al. [50] & 1625.44276 & 1797.70798 & 2096.8012 & 0.19 \\
Present Study (MGA) & 1621.246175 & 1739.156729 & 1992.961305 & 0.11 \\
\hline
\end{tabular}

Table 18. Friedman statistical test results for the hydrostatic thrust bearing design problem.

\begin{tabular}{ccc}
\hline Rankings & Algorithms & Mean of Ranks \\
\hline 1 & Present Study (MGA) & 1.5 \\
2 & Şahin et al. [74] & 2.5 \\
3 & Rao and Waghmare [75] & 3 \\
4 & Rao et al. [50] & 3 \\
\hline Chi-sq. & 3.6000 & \\
Prob > Chi-sq. & 0.3080 & \\
\hline
\end{tabular}

The optimum results of different metaheuristics in dealing with the ten-bar truss design problem are presented in Tables 19 and 20. By comparing the best results, it can be concluded that MGA is capable of outperforming other metaheuristics approaches. Until now, the best value obtained for this example was 529.25, which has been overcome by MGA with 529.12. This indicates the capability of MGA to provide remarkable results for some complex constrained design problems. 
Table 19. Comparison of the best solutions for the ten-bar truss design problem.

\begin{tabular}{cccccc}
\hline & Yu et al. [51] & $\begin{array}{c}\text { Lamberti and } \\
\text { Pappalettere [76] }\end{array}$ & $\begin{array}{c}\text { Baghlani and } \\
\text { Makiabadi [77] }\end{array}$ & $\begin{array}{c}\text { Kaveh and } \\
\text { Zolghadr [78] }\end{array}$ & $\begin{array}{c}\text { Present Study } \\
\text { (MGA) }\end{array}$ \\
\hline Best & 544.7 & 534.57 & 530.76 & 529.25 & 529.1204229 \\
$\mathrm{~A}_{1}$ & 36.380 & 35.148 & 35.494 & 39.569 & 36.76416 \\
$\mathrm{~A}_{2}$ & 12.941 & 13.169 & 14.777 & 16.740 & 16.29897 \\
$\mathrm{~A}_{3}$ & 35.764 & 37.69 & 36.203 & 34.361 & 37.94378 \\
$\mathrm{~A}_{4}$ & 18.314 & 19.556 & 15.387 & 12.994 & 16.51087 \\
$\mathrm{~A}_{5}$ & 3.002 & 1.087 & 0.6451 & 0.645 & 0.659 \\
$\mathrm{~A}_{6}$ & 5.433 & 4.844 & 4.5896 & 4.802 & 4.57489 \\
$\mathrm{~A}_{7}$ & 20.989 & 18.314 & 23.211 & 26.182 & 22.94023 \\
$\mathrm{~A}_{8}$ & 24.14 & 27.415 & 24.561 & 21.260 & 22.63185 \\
$\mathrm{~A}_{9}$ & 9.753 & 12.562 & 12.482 & 11.766 & 10.87892 \\
$\mathrm{~A}_{10}$ & 18.102 & 12.106 & 12.324 & 11.392 & 11.53643 \\
\hline
\end{tabular}

Table 20. Statistical results of the different method for the ten-bar truss bearing design problem.

\begin{tabular}{ccccc}
\hline Approaches & Best & Mean & Worst & Std-Dev \\
\hline Present Study (MGA) & 529.1204229 & 534.6843574 & 548.0179132 & 26.33651675 \\
\hline
\end{tabular}

The results of different methods for the rolling element bearing design problem are presented in Tables 21 and 22. It is clear that the best result of the MGA in this case is better than those of other approaches in the literature. Regarding the fact that this problem is a maximization optimization problem, MGA is also capable of providing remarkable statistical results.

Table 21. Comparison of the best solutions for the rolling element bearing design problem.

\begin{tabular}{ccc}
\hline & TLBO [50] & Present Study (MGA) * \\
\hline Best & $81,859.74$ & $83,912.87983$ \\
$\mathrm{D}_{\mathrm{m}}$ & 21.42559 & 125.0002787 \\
$\mathrm{D}_{\mathrm{b}}$ & 125.7191 & 21.87451192 \\
$\mathrm{Z}$ & 11 & 10.77706583 \\
$\mathrm{f}_{\mathrm{i}}$ & 0.515 & 0.515000822 \\
$\mathrm{f}_{0}$ & 0.515 & 0.515002993 \\
$\mathrm{~K}_{\mathrm{Dmin}}$ & 0.424266 & 0.405908353 \\
$\mathrm{~K}_{\mathrm{Dmax}}$ & 0.633948 & 0.65558802 \\
$\varepsilon$ & 0.3 & 0.300004155 \\
$\mathrm{e}$ & 0.068858 & 0.077544926 \\
$\zeta$ & 0.799498 & 0.6 \\
\hline
\end{tabular}

* This problem is a maximization problem.

Table 22. Statistical results of different approaches for the rolling element bearing design problem.

\begin{tabular}{ccccc}
\hline Approaches & Best & Mean & Worst & Std-Dev \\
\hline TLBO [50] & $81,859.74$ & $81,438.987$ & $80,807.8551$ & 0.66 \\
Present Study (MGA) & $83,912.87983$ & $83,892.25647$ & $83,711.21317$ & 23.65841 \\
\hline
\end{tabular}

Table A17 [46,79-81] and Table 23 display the comparative and statistical optimization results of multiple optimization algorithms and MGA in dealing with the gear train design problem. It is obvious that MGA outranks the other optimization algorithms, Specifically, MGA obtained a perfect best of zero, which has not been obtained by other metaheuristics, confirming the capability of MGA to yield the lowest possible value in this case. The Friedman statistical test results are also presented in Table A18 for comparative purposes. 
Table 23. Statistical results of different approaches for the gear train design problem.

\begin{tabular}{ccccc}
\hline Approaches & Best & Mean & Worst & Std-Dev \\
\hline Gandomi et al. [46] & $2.7009 \times 10^{-12}$ & $1.9841 \times 10^{-9}$ & $2.3576 \times 10^{-9}$ & $3.5546 \times 10^{-9}$ \\
Loh and Papalambros [79] & $2.7 \times 10^{-12}$ & $2.7 \times 10^{-12}$ & $2.7 \times 10^{-12}$ & $2.2122 \times 10^{-28}$ \\
Wang et al. [82] (CPKH) & $2.22 \times 10^{-16}$ & $2.22 \times 10^{-16}$ & $8.5 \times 10^{-9}$ & $7.96 \times 10^{-22}$ \\
Wang et al. [82] (ABC) & $2.92 \times 10^{-15}$ & $3.18 \times 10^{-15}$ & $8.5 \times 10^{-9}$ & $9.81 \times 10^{-10}$ \\
Present Study (MGA) & $1.06 \times 10^{-19}$ & $7.69 \times 10^{-14}$ & $7.62 \times 10^{-13}$ & $1.78 \times 10^{-13}$ \\
\hline
\end{tabular}

CPKH: Chaotic Particle Swarm Krill Herd.

Considering the steel I-shaped beam as one of the most well-formulated design problems, the final and statistical optimization results of multiple metaheuristics are presented in Tables 24 and 25, respectively. By comparing these optimum results, MGA outranked all other well-known algorithms that have been reported recently.

Table 24. Comparison of the best solutions for the steel I-shaped beam design problem.

\begin{tabular}{cccccc}
\hline & ARSM [83] & I-ARSM [83] & MATLAB [83] & CS [46] & $\begin{array}{c}\text { Present Study } \\
\text { (MGA) }\end{array}$ \\
\hline Best & 0.0157 & 0.131 & 0.0131 & 0.0130747 & 0.013074119 \\
$\mathrm{~h}$ & 80 & 79.99 & 80 & 80 & 79.9999992 \\
$\mathrm{~b}$ & 37.05 & 48.42 & 50 & 50 & 49.9999985 \\
$\mathrm{t}_{\mathrm{w}}$ & 1.71 & 0.9 & 0.9 & 0.9 & 0.9 \\
$\mathrm{t}_{\mathrm{f}}$ & 2.31 & 2.4 & 2.32 & 2.3216715 & 2.321792333 \\
\hline
\end{tabular}

ARSM: Adaptive Response Surface Method; I-ARMS: Improved Adaptive Response Surface Method; MATLAB Matrix Laboratory Optimization Approach.

Table 25. Statistical results of different approaches for the steel I-shaped beam design problem.

\begin{tabular}{ccccc}
\hline Approaches & Best & Mean & Worst & Std-Dev \\
\hline CS [46] & 0.0130747 & 0.0132165 & 0.01353646 & 0.0001345 \\
Present Study (MGA) & 0.013074119 & 0.013074141 & 0.013074291 & $3.86 \times 10^{-8}$ \\
\hline
\end{tabular}

The final results of different metaheuristics for the piston lever design problem, a frequently occurring optimization problem, are presented in Table A19. The statistical results, including the best, mean, and worst fitness values alongside standard deviation, are presented in Table 26 for comparative purposes. Based on the results, MGA is capable of providing better statistical (mean, worst, and standard deviation of the results) and greatly outranked the other algorithms in terms of the best results. The Friedman statistical test results are also presented in Table A20 for comparative purposes.

Table 26. Statistical results of different approaches for the piston lever design problem.

\begin{tabular}{ccccc}
\hline Approaches & Best & Mean & Worst & Std-Dev \\
\hline HPSO [46] & 162 & 187 & 197 & 13.4 \\
GA [46] & 161 & 185 & 216 & 18.2 \\
DE [46] & 159 & 187 & 199 & 14.2 \\
CSA [46] & 8.4271 & 40.2319 & 168.5920 & 59.0552 \\
Present Study (MGA) & 8.413406652 & 32.4688925 & 167.4732134 & 29.96370439 \\
\hline
\end{tabular}

HPSO: Hybrid Particle Swarm Optimization.

Considering the cantilever beam engineering design problem, the optimization results of the different optimization algorithms are all presented in Tables 27 and 28. By comparing the best results of these methods, it can be concluded that MGA is capable of achieving 
better results. According to the literature, recently-developed algorithms can yield 1.34, at best, for this example. Herein, we found that MGA is capable of providing even better result (1.33997) by conducting a better searching procedure. The statistical results of other optimization algorithms are not reported in the literature; thus, the remarkable results of MGA are beneficial for future works.

Table 27. Comparison of the best solutions for the cantilever beam design problem.

\begin{tabular}{cccccc}
\hline & MMA [46] & GCA-I [46] & GCA-II [46] & CSA [46] & $\begin{array}{c}\text { Present Study } \\
\text { (MGA) }\end{array}$ \\
\hline Best & 1.34 & 1.34 & 1.34 & 1.33999 & 1.339975661 \\
$\mathrm{x}_{1}$ & 6.01 & 6.01 & 6.01 & 6.0089 & 6.011660964 \\
$\mathrm{x}_{2}$ & 5.3 & 5.3 & 5.3 & 5.3049 & 5.315676194 \\
$\mathrm{x}_{3}$ & 4.49 & 4.49 & 4.49 & 4.5023 & 4.510681877 \\
$\mathrm{x}_{4}$ & 3.49 & 3.49 & 3.49 & 3.5077 & 3.485698713 \\
$\mathrm{x}_{5}$ & 2.15 & 2.15 & 2.15 & 2.1504 & 2.150251174 \\
\hline
\end{tabular}

MMA: Method of Moving Asymptotes; GCA: Generalized Convex Approximation.

Table 28. Statistical results of the MGA method for the cantilever beam design problem.

\begin{tabular}{ccccc}
\hline Approaches & Best & Mean & Worst & Std-Dev \\
\hline Present Study (MGA) & 1.339975661 & 1.340052681 & 1.340201166 & $6.99 \times 10^{-5}$ \\
\hline
\end{tabular}

By comparing the $p$-values of the Friedman statistical test which are presented in the table of results by Chi-sq., it is concluded that for the piston lever design example, the lowest $p$-values is determined which demonstrates the fact that for this example, there are noticeable difference between the results of different approaches. However, the $p$-values of other examples are also near a mean of 9 which represents the stability of the conducted optimization runs and the statistical tests (Figure 11).

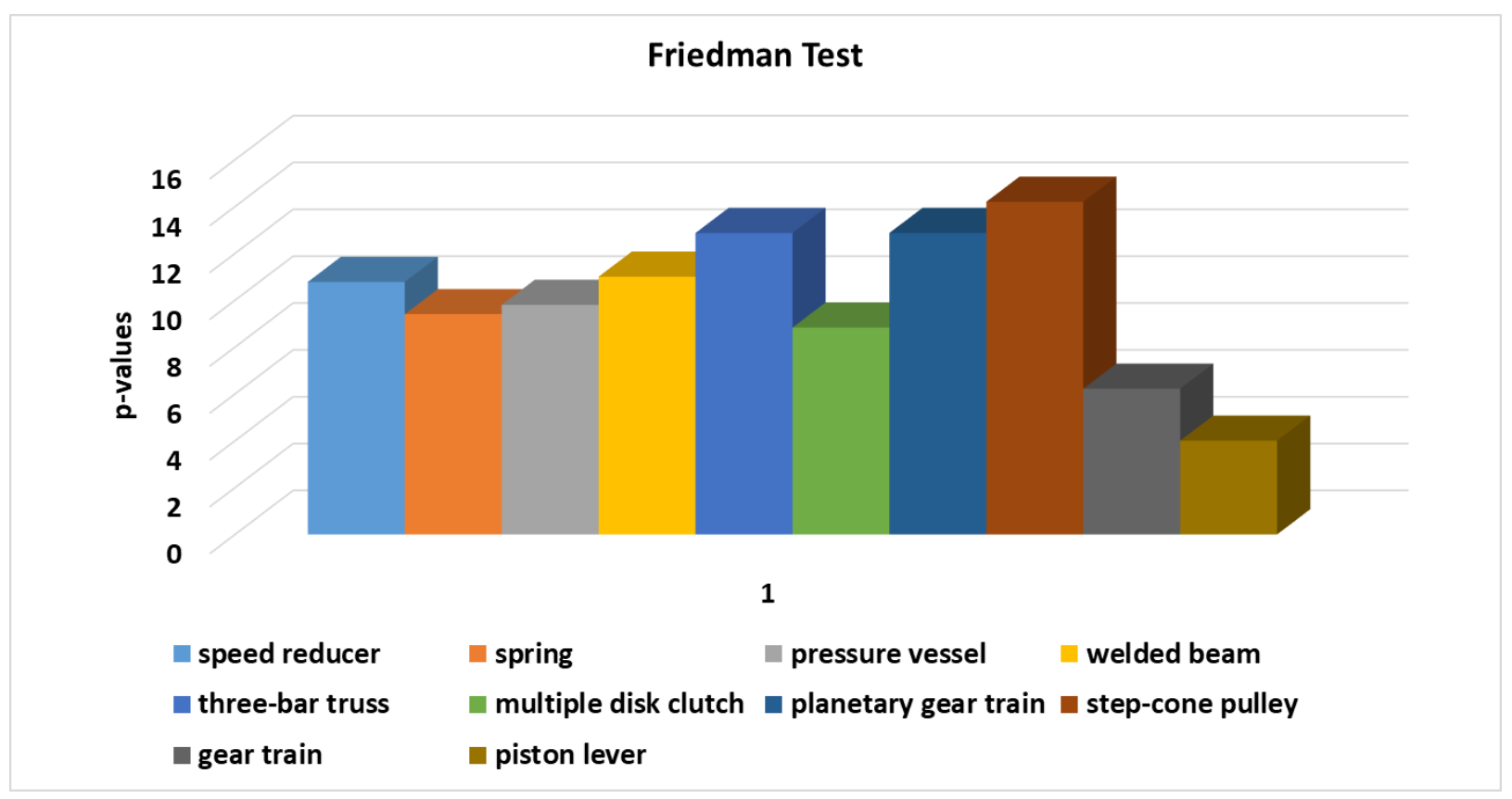

Figure 11. Comparison of Friedman's p-vales for different design examples. 


\section{Conclusions}

In this paper, the Material Generation Algorithm (MGA) is presented as a new metaheuristic for different applications and various optimization problems. In this regard, 25 constrained design problems were considered to evaluate MGA, including 10 mathematically-constrained problems presented by the Competitions on Evolutionary Computation (CEC 2017) and 15 well-known engineering design problems. For comparative purposes, the best results of different metaheuristic algorithms, such as state-of-the-art metaheuristics from CEC 2017, were selected for comparative purposes. Considering the results of MGA in dealing with the mathematical problems, it should be noted that this algorithm is capable of providing very competitive results in different dimensions. In addition, MGA yielded very impressive results in all of constrained engineering design problems compared to the previously reported algorithms. Specifically, the highest difference of about $24 \%$ between the best results of MGA and the best results reported thus far in the literature was found for the multiple disk clutch brake engineering design problem. For the three-bar truss design problem, MGA can provide very competitive results and, importantly, nearly the best results reported thus far. For the tension or compression spring, pressure vessel and rolling element bearing problems, the best results were higher for MGA than the best reported results.

While the proposed MGA has been proven to be a powerful method, different applications of this method are suggested for future research. It should be mentioned that the capability of this optimization approach can be controlled in dealing with some complex real-world and even computationally-expensive optimization problems. In addition, some other challenges, such as improving the general formulation of this method and hybridizing with other approaches, should be investigated properly.

Author Contributions: Conceptualization, S.T.; methodology, S.T. and M.A.; software, S.T. and M.A.; Validation, M.A. and S.T.; formal analysis, M.A.; investigation, S.T., M.A. and A.H.G.; resources, S.T., M.A. and A.H.G.; data curation, A.H.G.; writing—original draft preparation, M.A.; writing-review and editing, S.T. and A.H.G.; visualization, M.A.; supervision, S.T.; project administration, S.T.; funding acquisition, S.T., M.A. and A.H.G. All authors have read and agreed to the published version of the manuscript.

Funding: The APC was funded by University of Technology Sydney Internal Fund for A.H. Gandomi.

Institutional Review Board Statement: Not applicable.

Informed Consent Statement: Not applicable.

Data Availability Statement: The Matlab implementation of MGA is accessible at: www.mathworks. com/matlabcentral/fileexchange/92065-material-generation-algorithm-mga (accessed on 3 May 2021).

Acknowledgments: This research was partially supported by the University of Tabriz, grant number 1615 and the APC was funded by University of Technology Sydney Internal Fund for A.H. Gandomi.

Conflicts of Interest: The authors declare no conflict of interest.

\section{Appendix A}

Table A1. Comparison of the best solutions for the speed reducer design problem.

\begin{tabular}{cccccc}
\hline & $\begin{array}{c}\text { Montes et al. } \\
{[54]}\end{array}$ & $\begin{array}{c}\text { Akhtar et al. } \\
{[55]}\end{array}$ & $\begin{array}{c}\text { Gandomi et al. } \\
{[46]}\end{array}$ & $\begin{array}{c}\text { Zhang et al. } \\
{[56]}\end{array}$ & $\begin{array}{c}\text { Present Study } \\
\text { (MGA) }\end{array}$ \\
\hline Best & 3025.005 & 3008.08 & 3000.9810 & 2994.471066 & 2994.438869 \\
$\mathrm{~b}$ & 3.506163 & 3.506122 & 3.5015 & 3.5 & 3.500007956 \\
$\mathrm{~m}$ & 0.700831 & 0.700006 & 0.7000 & 0.7 & 0.700000656 \\
$\mathrm{z}$ & 17 & 17 & 17.0000 & 17 & 17.00000081 \\
$\mathrm{l}_{1}$ & 7.460181 & 7.549126 & 7.6050 & 7.3 & 7.300541927 \\
\hline
\end{tabular}


Table A1. Cont.

\begin{tabular}{lccccc}
\hline & $\begin{array}{c}\text { Montes et al. } \\
{[54]}\end{array}$ & $\begin{array}{c}\text { Akhtar et al. } \\
{[\text { [55] }}\end{array}$ & $\begin{array}{c}\text { Gandomi et al. } \\
{[46]}\end{array}$ & $\begin{array}{c}\text { Zhang et al. } \\
{[56]}\end{array}$ & $\begin{array}{c}\text { Present Study } \\
\text { (MGA) }\end{array}$ \\
\hline $\mathrm{l}_{2}$ & 7.962143 & 7.85933 & 7.8181 & 7.7153199115 & 7.715357693 \\
$\mathrm{~d}_{1}$ & 3.3629 & 3.365576 & 3.3520 & 3.3502146661 & 3.350542391 \\
$\mathrm{~d}_{2}$ & 5.3090 & 5.289773 & 5.2875 & 5.2866544650 & 5.28665793 \\
$\mathrm{~g}_{1}(\mathrm{x})$ & -0.0777 & -0.0755 & -0.0743 & -0.0739152 & -2.155122277 \\
$\mathrm{~g}_{2}(\mathrm{x})$ & -0.2013 & -0.1994 & -0.1983 & -0.1979985 & -98.13710222 \\
$\mathrm{~g}_{3}(\mathrm{x})$ & -0.4741 & -0.4562 & -0.4349 & -0.9999967 & -1.924273761 \\
$\mathrm{~g}_{4}(\mathrm{x})$ & -0.8971 & -0.8994 & -0.9008 & -0.9999995 & -18.30969834 \\
$\mathrm{~g}_{5}(\mathrm{x})$ & -0.0110 & -0.0132 & -0.0011 & -0.6668526 & -0.000437152 \\
$\mathrm{~g}_{6}(\mathrm{x})$ & -0.0125 & -0.0017 & -0.0004 & -0.0000000 & -0.001666474 \\
$\mathrm{~g}_{7}(\mathrm{x})$ & -0.7022 & -0.7025 & -0.7025 & -0.7025000 & -28.09998829 \\
$\mathrm{~g}_{8}(\mathrm{x})$ & -0.0006 & -0.0017 & -0.0004 & -0.0000000 & $-6.68 \times 10^{-6}$ \\
$\mathrm{~g}_{9}(\mathrm{x})$ & -0.5831 & -0.5826 & -0.5832 & -0.5833333 & -6.999993318 \\
$\mathrm{~g}_{10}(\mathrm{x})$ & -0.0691 & -0.0796 & -0.0890 & -0.0513257 & -0.374728341 \\
$\mathrm{~g}_{11}(\mathrm{x})$ & -0.0279 & -0.0179 & -0.0130 & -0.0000000 & $-3.40 \times 10^{-05}$ \\
\hline
\end{tabular}

Table A2. Friedman statistical test results for the speed reducer design problem.

\begin{tabular}{ccc}
\hline Rankings & Algorithms & Mean of Ranks \\
\hline 1 & Present Study (MGA) & 1.50 \\
2 & Zhang et al. [56] & 2.00 \\
3 & Gandomi et al. [46] & 3.00 \\
4 & Akhtar et al. [55] & 3.87 \\
5 & Montes et al. [54] & 4.62 \\
\hline Chi-sq. & & \\
Prob > Chi-sq. & 0.0291 & \\
\hline
\end{tabular}

Table A3. Comparison of the best solutions for the tension or compression spring design problem.

\begin{tabular}{clcccc}
\hline & $\begin{array}{c}\text { Coello } \\
\text { [57] }\end{array}$ & $\begin{array}{c}\text { Ray and } \\
\text { Liew [58] }\end{array}$ & Han et al. [59] & $\begin{array}{c}\text { Gandomi et al. } \\
\text { [45] }\end{array}$ & $\begin{array}{c}\text { Present Study } \\
\text { (MGA) }\end{array}$ \\
\hline Best & 0.01270478 & 0.0126692 & 0.01266534 & 0.01266522 & 0.01266523 \\
d & 0.051480 & 0.052160 & 0.0516800 & 0.05169 & 0.051689061 \\
D & 0.351661 & 0.368159 & 0.3565001 & 0.35673 & 0.35671774 \\
$\mathrm{~N}$ & 11.632201 & 10.648442 & 11.3018335 & 11.2885 & 11.28896576 \\
$\mathrm{~g}_{1}(\mathrm{x})$ & -0.003337 & $-7.45 \times 10^{-9}$ & $-6.218 \times 10^{-6}$ & 0 & 0 \\
$\mathrm{~g}_{2}(\mathrm{x})$ & -0.000110 & $-3.68 \times 10^{-9}$ & $-1.691 \times 10^{-6}$ & 0 & 0 \\
$\mathrm{~g}_{3}(\mathrm{x})$ & -4.026318 & -4.075805 & -4.0533150 & -4.0538 & -4.05378563 \\
$\mathrm{~g}_{4}(\mathrm{x})$ & -0.731239 & -0.719787 & -0.7278799 & -0.7277 & -0.7277288 \\
\hline
\end{tabular}

Table A4. Friedman statistical test results for the tension or compression spring design problem.

\begin{tabular}{ccc}
\hline Rankings & Algorithms & Mean of Ranks \\
\hline 1 & Present Study (MGA) & 1.25 \\
2 & Han et al. [59] & 2.25 \\
3 & Coello [57] & 3.50 \\
4 & Ray and Liew [58] & 4.00 \\
5 & Gandomi et al. [45] & 4.00 \\
\hline Chi-sq. & & \\
Prob > Chi-sq. & & \\
\hline
\end{tabular}


Table A5. Comparison of the best solutions for the pressure vessel design problem.

\begin{tabular}{cccccc}
\hline & $\begin{array}{c}\text { He and } \\
\text { Wang [60] }\end{array}$ & Coelho [61] & $\begin{array}{c}\text { Mezura- } \\
\text { Montes and } \\
\text { Coello [62] }\end{array}$ & $\begin{array}{c}\text { Coello and } \\
\text { Montes [63] }\end{array}$ & $\begin{array}{c}\text { Present } \\
\text { Study } \\
\text { (MGA) }\end{array}$ \\
\hline Best & 6061.0777 & 6059.7208 & 6059.7456 & 6059.9463 & 6059.714350 \\
$\mathrm{~T}_{\mathrm{s}}$ & 0.8125 & 0.8125 & 0.8125 & 0.8125 & 0.8125 \\
$\mathrm{~T}_{\mathrm{h}}$ & 0.4375 & 0.4375 & 0.4375 & 0.4375 & 0.4375 \\
$\mathrm{R}$ & 42.0913 & 42.0984 & 42.098087 & 42.097398 & 42.0984 \\
$\mathrm{~L}$ & 176.7465 & 176.6372 & 176.640518 & 176.654050 & 176.6366 \\
$\mathrm{~g}_{1}(\mathrm{x})$ & $-1.37 \times 10^{-6}$ & $-8.79 \times 10^{-7}$ & $-6.92 \times 10^{-6}$ & $-2.02 \times 10^{-5}$ & 0 \\
$\mathrm{~g}_{2}(\mathrm{x})$ & $-3.59 \times 10^{-4}$ & $-3.58 \times 10^{-2}$ & -0.03588 & -0.03589 & -0.0359 \\
$\mathrm{~g}_{3}(\mathrm{x})$ & -118.7687 & -0.2179 & 2.903372 & -24.8998 & 0 \\
$\mathrm{~g}_{4}(\mathrm{x})$ & -63.2535 & -63.3628 & -63.3595 & -63.346 & -63.3634 \\
\hline
\end{tabular}

Table A6. Friedman statistical test results for the pressure vessel design problem.

\begin{tabular}{|c|c|c|}
\hline Rankings & Algorithms & Mean of Ranks \\
\hline 1 & Present Study (MGA) & 1 \\
\hline 2 & He and Wang [60] & 2.75 \\
\hline 3 & \multirow{2}{*}{$\begin{array}{c}\text { Coello and Montes [63] } \\
\text { Coelho [61] }\end{array}$} & 3.25 \\
\hline 4 & & 4.00 \\
\hline 5 & $\begin{array}{c}\text { Mezura-Montes and Coello } \\
{[62]}\end{array}$ & 4.00 \\
\hline $\begin{array}{c}\text { Chi-sq. } \\
\text { Prob > Chi-sq. }\end{array}$ & \multicolumn{2}{|c|}{$\begin{array}{l}9.8000 \\
0.0439\end{array}$} \\
\hline
\end{tabular}

Table A7. Comparison of the best solutions for the welded beam design problem.

\begin{tabular}{cccccc}
\hline & $\begin{array}{c}\text { Huang et al. } \\
{[64]}\end{array}$ & $\begin{array}{c}\text { Eskandar et al. } \\
{[65]}\end{array}$ & Guedria [66] & $\begin{array}{c}\text { Han et al. } \\
{[59]}\end{array}$ & $\begin{array}{c}\text { Present } \\
\text { Study } \\
\text { (MGA) }\end{array}$ \\
\hline Best & 1.733461 & 1.724856 & 1.724852 & 1.6956397 & 1.672966512 \\
$\mathrm{~h}$ & 0.203137 & 0.205728 & 0.205730 & 0.20532536 & 0.198957505 \\
1 & 3.542998 & 3.470522 & 3.470489 & 3.26035648 & 3.341955765 \\
$\mathrm{t}$ & 9.033498 & 9.036620 & 9.036624 & 9.03664424 & 9.187291977 \\
$\mathrm{~b}$ & 0.206179 & 0.205729 & 0.205730 & 0.20572991 & 0.199190532 \\
$\mathrm{~g}_{1}(\mathrm{x})$ & -44.57856 & -0.034128 & $-1.05 \times 10^{-10}$ & -0.10520197 & -20.76244473 \\
$\mathrm{~g}_{2}(\mathrm{x})$ & -44.66353 & $-3.49 \times 10^{-5}$ & $-6.91 \times 10^{-10}$ & -0.17417862 & -23.09392302 \\
$\mathrm{~g}_{3}(\mathrm{x})$ & -0.003042 & $-1.19 \times 10^{-6}$ & $-7.66 \times 10^{-15}$ & -4.04330102 & -0.000233027 \\
$\mathrm{~g}_{4}(\mathrm{x})$ & -3.423726 & -3.432980 & -3.432984 & -3.45179021 & -3.469028817 \\
$\mathrm{~g}_{5}(\mathrm{x})$ & -0.078137 & -0.080728 & -0.080730 & -0.08032536 & -0.073957505 \\
$\mathrm{~g}_{6}(\mathrm{x})$ & -0.235557 & -0.235540 & -0.235540 & -0.22831066 & -0.05415088 \\
$\mathrm{~g}_{7}(\mathrm{x})$ & -38.02826 & -0.013503 & $-5.80 \times 10^{-10}$ & -0.03397937 & -30.47032014 \\
\hline
\end{tabular}

Table A8. Friedman statistical test results for the welded beam design problem.

\begin{tabular}{ccc}
\hline Rankings & Algorithms & Mean of Ranks \\
\hline 1 & Present Study (MGA) & 1.50 \\
2 & Guedria [66] & 2.25 \\
3 & Han et al. [59] & 3.25 \\
4 & Eskandar et al. [65] & 3 \\
\hline
\end{tabular}


Table A8. Cont.

\begin{tabular}{ccc}
\hline Rankings & Algorithms & Mean of Ranks \\
\hline 5 & Huang et al. [64] & 5 \\
\hline Chi-sq. & & 11.0000 \\
Prob $>$ Chi-sq. & 0.0266 & \\
\hline
\end{tabular}

Table A9. Comparison of the best solutions for the three-bar truss design problem.

\begin{tabular}{lccccc}
\hline & $\begin{array}{c}\text { Gandomi et al. } \\
{[46]}\end{array}$ & $\begin{array}{c}\text { Ray and Liew } \\
{[58]}\end{array}$ & Zhang et al. [56] & Grag [67] & $\begin{array}{c}\text { Present } \\
\text { Study } \\
\text { (MGA) }\end{array}$ \\
\hline Best & 263.97156 & 263.8958466 & 263.8958434 & 263.8958433 & 263.8958433 \\
$\mathrm{~A}_{1}$ & 0.78867 & 0.7886210370 & 0.7886751359 & 0.788676171219 & 0.788675136 \\
$\mathrm{~A}_{2}$ & 0.40902 & 0.4084013340 & 0.4082482868 & 0.408245358456 & 0.408248288 \\
$\mathrm{~g}_{1}(\mathrm{x})$ & -0.00029 & $-8.275 \times 10^{-9}$ & $-2.104 \times 10^{-11}$ & $-1.587 \times 10^{-13}$ & 0 \\
$\mathrm{~g}_{2}(\mathrm{x})$ & -0.00029 & -1.46392765 & -1.46410161 & -1.4641049 & -1.464101618 \\
$\mathrm{~g}_{3}(\mathrm{x})$ & -0.73176 & -0.536072358 & -0.5358983 & -0.535895 & -0.535898382 \\
\hline
\end{tabular}

Table A10. Friedman statistical test results for the three-bar truss design problem.

\begin{tabular}{ccc}
\hline Rankings & Algorithms & Mean of Ranks \\
\hline 1 & Present Study (MGA) & 1.75 \\
2 & Grag [67] & 1.87 \\
3 & Zhang et al. [56] & 2.37 \\
4 & Ray and Liew [58] & 4.25 \\
5 & Gandomi et al. [46] & 4.75 \\
\hline Chi-sq. & \multicolumn{1}{|}{} \\
Prob > Chi-sq. & 0.0119 \\
\hline
\end{tabular}

Table A11. Comparison of the best solutions for the multiple disk clutch brake design problem.

\begin{tabular}{cccccc}
\hline & $\begin{array}{c}\text { Deb and } \\
\text { Srinivasan } \\
{[68]}\end{array}$ & $\begin{array}{c}\text { Eskandar et al. } \\
{[65]}\end{array}$ & $\begin{array}{c}\text { Rao et al. } \\
{[50]}\end{array}$ & $\begin{array}{c}\text { Ferreira et al. } \\
{[47]}\end{array}$ & $\begin{array}{c}\text { Present } \\
\text { Study } \\
\text { (MGA) }\end{array}$ \\
\hline Best & 0.4704 & 0.313656 & 0.313656611 & 0.313656 & 0.235242467 \\
$\mathrm{r}_{1}$ & 70 & 70 & 70 & 70 & 70.00000008 \\
$\mathrm{r}_{0}$ & 90 & 90 & 90 & 90 & 90.0000003 \\
$\mathrm{t}$ & 1.5 & 1 & 1 & 1 & 1.000000013 \\
$\mathrm{~F}$ & 1000 & 910 & 810 & 830 & 865.6907633 \\
$\mathrm{Z}$ & 3 & 3 & 3 & 3 & 2.00000004 \\
$\mathrm{~g}_{1}(\mathrm{x})$ & 0 & 0 & 0 & 0 & $-2.18 \times 10^{-7}$ \\
$\mathrm{~g}_{2}(\mathrm{x})$ & -22 & -24 & -24 & -24 & -25.4999999 \\
$\mathrm{~g}_{3}(\mathrm{x})$ & -0.9005 & -0.909480 & -0.91942781 & -0.917438 & -0.913888149 \\
$\mathrm{~g}_{4}(\mathrm{x})$ & -9.7906 & -9.809429 & -9830.371094 & -9.826183 & -9.985383395 \\
$\mathrm{~g}_{5}(\mathrm{x})$ & -7.8947 & -7.894696 & -7894.69659 & -7.894697 & -9.830260243 \\
$\mathrm{~g}_{6}(\mathrm{x})$ & -3.3527 & -2.231421 & -0.702013203 & -0.173855 & -14.98276443 \\
$\mathrm{~g}_{7}(\mathrm{x})$ & -60.6250 & -49.768749 & -37706.25 & -40.118750 & -83479.16052 \\
$\mathrm{~g}_{8}(\mathrm{x})$ & -11.6473 & -12.768578 & -14.2979868 & -14.826145 & -0.017235569 \\
\hline
\end{tabular}


Table A12. Friedman statistical test results for the multiple disk clutch brake design problem.

\begin{tabular}{ccc}
\hline Rankings & Algorithms & Mean of Ranks \\
\hline 1 & Present Study (MGA) & 1.5 \\
2 & Ferreira et al. [47] & 2.12 \\
3 & Eskandar et al. [65] & 2.37 \\
4 & Rao et al. [50] & 4.00 \\
\hline Chi-sq. & 8.8378 & \\
Prob > Chi-sq. & 0.0315 & \\
\hline
\end{tabular}

Table A13. Comparison of the best solutions for the planetary gear train design problem.

\begin{tabular}{ccc}
\hline & Savsani and Savsani [48] & Present Study (MGA) \\
\hline Best & 0.525588 & 0.52325 \\
$\mathrm{~N}_{1}$ & 34 & 40 \\
$\mathrm{~N}_{2}$ & 25 & 21 \\
$\mathrm{~N}_{3}$ & 33 & 14 \\
$\mathrm{~N}_{4}$ & 32 & 19 \\
$\mathrm{~N}_{5}$ & 23 & 17 \\
$\mathrm{~N}_{6}$ & 116 & 69 \\
$\mathrm{P}$ & 4 & 3 \\
$\mathrm{~m}_{1}$ & 2.5 & 2 \\
$\mathrm{~m}_{2}$ & 1.75 & 3 \\
\hline
\end{tabular}

Table A14. Friedman statistical test results for the planetary gear train design problem.

\begin{tabular}{ccc}
\hline Rankings & Algorithms & Mean of Ranks \\
\hline 1 & Present Study (MGA) & 2.50 \\
2 & Zhang et al. [70] & 2.50 \\
3 & Savsani and Savsani [48] & 3.00 \\
4 & Rao and Savsani [69] (ABC) & 3.00 \\
5 & Rao and Savsani [69] (PSO) & 4.00 \\
\hline Chi-sq. & 12.8700 \\
Prob > Chi-sq. & 0.0119 \\
\hline
\end{tabular}

Table A15. Comparison of the best solutions for the step-cone pulley design problem.

\begin{tabular}{cccccc}
\hline & TLBO [50] & WOA [44] & WCA [44] & MBA [44] & $\begin{array}{c}\text { Present Study } \\
\text { (MGA) }\end{array}$ \\
\hline Best & 16.63451 & 16.6345213 & 16.63450849 & 16.6345078 & 16.18595608 \\
$\mathrm{~d}_{1}$ & 40 & 40 & 40 & 40 & 38.53034981 \\
$\mathrm{~d}_{2}$ & 54.7643 & 54.764326 & 54.764300 & 54.764300 & 53.04151483 \\
$\mathrm{~d}_{3}$ & 73.01318 & 54.764326 & 54.764300 & 54.764300 & 70.67294075 \\
$\mathrm{~d}_{4}$ & 73.01318 & 54.764326 & 54.764300 & 88.428419 & 84.71470998 \\
$\mathrm{w}$ & 73.01318 & 85.986297 & 54.764300 & 85.986242 & 90 \\
\hline
\end{tabular}

WOA: Whale Optimization Algorithm; WCA: Water Cycle Algorithm; MBA: Mine Blast Algorithm. 
Table A16. Friedman statistical test results for the step-cone pulley design problem.

\begin{tabular}{ccc}
\hline Rankings & Algorithms & Mean of Ranks \\
\hline 1 & Present Study (MGA) & 1.00 \\
2 & MBA [44] & 2.00 \\
3 & WCA [44] & 3.25 \\
4 & TLBO [50] & 4.25 \\
5 & WOA [44] & 4.50 \\
\hline Chi-sq. & 14.2000 \\
Prob > Chi-sq. & 0.0067 \\
\end{tabular}

Table A17. Comparison of the best solutions for the gear train design problem.

\begin{tabular}{cccccc}
\hline & $\begin{array}{c}\text { Gandomi et al. } \\
{[46]}\end{array}$ & $\begin{array}{c}\text { Loh and Pa- } \\
\text { palambros } \\
{[79]}\end{array}$ & $\begin{array}{c}\text { Kannan and } \\
\text { Kramer [80] }\end{array}$ & $\begin{array}{c}\text { Sandgren } \\
{[81]}\end{array}$ & $\begin{array}{c}\text { Present } \\
\text { Study } \\
\text { (MGA) }\end{array}$ \\
\hline Best & $2.701 \times 10^{-12}$ & $2.7 \times 10^{-12}$ & $2.146 \times 10^{-8}$ & $5.712 \times 10^{-6}$ & $1.06 \times 10^{-19}$ \\
$\mathrm{Z}_{\mathrm{d}}$ & 19 & 19 & 13 & 18 & 27.32076302 \\
$\mathrm{Z}_{\mathrm{b}}$ & 16 & 16 & 15 & 22 & 13.75530503 \\
$\mathrm{Z}_{\mathrm{a}}$ & 43 & 43 & 33 & 45 & 48.25305913 \\
$\mathrm{Z}_{\mathrm{f}}$ & 49 & 49 & 41 & 60 & 53.98015133 \\
\hline
\end{tabular}

Table A18. Friedman statistical test results for the gear train design problem.

\begin{tabular}{ccc}
\hline Rankings & Algorithms & Mean of Ranks \\
\hline 1 & Present Study (MGA) & 2.00 \\
2 & Wang et al. [82] (CPKH) & 2.37 \\
3 & Loh and Papalambros [79] & 2.75 \\
4 & Wang et al. [82] (ABC) & 3.37 \\
5 & Gandomi et al. [46] & 4.50 \\
\hline Chi-sq. & & \\
Prob > Chi-sq. & 0.2278 & \\
\hline
\end{tabular}

Table A19. Comparison of the best solutions for the piston lever design problem.

\begin{tabular}{ccc}
\hline & CSA [46] & Present Study (MGA) \\
\hline Best & 8.4271 & 8.413406652 \\
H & 0.05 & 0.05 \\
B & 2.043 & 2.041637535 \\
X & 120 & 120 \\
D & 4.0851 & 4.083080224 \\
\hline
\end{tabular}

Table A20. Friedman statistical test results for the piston lever design problem.

\begin{tabular}{ccc}
\hline Rankings & Algorithms & Mean of Ranks \\
\hline 1 & Present Study (MGA) & 1.75 \\
2 & CSA [46] & 2.75 \\
3 & HPSO [46] & 3.75 \\
4 & GA [46] & 3.75 \\
5 & DE [46] & 3.75 \\
\hline Chi-sq. & & \\
Prob > Chi-sq. & 4.0000 & \\
\hline
\end{tabular}




\section{References}

1. Glover, F. Future paths for integer programming and links to artificial intelligence. Comput. Oper. Res. 1986, 13, 533-549. [CrossRef]

2. Sörensen, K.; Sevaux, M.; Glover, F. A History of Metaheuristics; Handbook of heuristics. arXiv 2017, arXiv:1704.00853.

3. Moscato, P. On Evolution, Search, Optimization, Genetic Algorithms and Martial Arts: Towards Memetic Algorithms. Caltech Concurr. Comput. Program C3P Rep. 1989, 826, 1989.

4. Holland, J.H. Adaptation in Natural and Artificial Systems: An Introductory Analysis with Applications to Biology, Control, and Artificial Intelligence; MIT Press: Cambridge, MA, USA, 1992.

5. Koza, J.R.; Koza, J.R. Genetic Programming: On the Programming of Computers by Means of Natural Selection; MIT Press: Cambridge, MA, USA, 1992.

6. Storn, R.; Price, K. Differential evolution-A simple and efficient heuristic for global optimization over continuous spaces. J. Glob. Optim. 1997, 11, 341-359. [CrossRef]

7. Beyer, H.G.; Schwefel, H.P. Evolution strategies-A comprehensive introduction. Nat. Comput. 2002, 1, 3-52. [CrossRef]

8. Simon, D. Biogeography-based optimization. IEEE Trans. Evol. Comput. 2008, 12, 702-713. [CrossRef]

9. Eberhart, R.; Kennedy, J. A New Optimizer Using Particle Swarm Theory. In Proceedings of the Sixth International Symposium on Micro Machine and Human Science, Nagoya, Japan, 4-6 October 1995; pp. 39-43.

10. Dorigo, M.; Maniezzo, V.; Colorni, A. Ant system: Optimization by a colony of cooperating agents. IEEE Trans. Syst. Man Cybern. Part B Cybern. 1996, 26, 29-41. [CrossRef] [PubMed]

11. Basturk, B. An artificial bee colony (ABC) algorithm for numeric function optimization. In Proceedings of the IEEE Swarm Intelligence Symposium, Indianapolis, IN, USA, 12 May 2006.

12. Chu, S.C.; Tsai, P.W.; Pan, J.S. Cat Swarm Optimization. In Pacific Rim International Conference on Artificial Intelligence; Springer: Berlin/Heidelberg, Germany, 2006; pp. 854-858.

13. Yang, X.S. Nature-Inspired Metaheuristic Algorithms; Luniver Press: Bristol, UK, 2010.

14. Gandomi, A.H.; Alavi, A.H. Krill herd: A new bio-inspired optimization algorithm. Commun. Nonlinear Sci. Numer. Simul. 2012, 17, 4831-4845. [CrossRef]

15. Kirkpatrick, S.; Gelatt, C.D.; Vecchi, M.P. Optimization by simulated annealing. Science 1983, 220, 671-680. [CrossRef]

16. Geem, Z.W.; Kim, J.H.; Loganathan, G.V. A new heuristic optimization algorithm: Harmony search. Simulation 2001, 76, 60-68. [CrossRef]

17. Erol, O.K.; Eksin, I. A new optimization method: Big bang-big crunch. Adv. Eng. Softw. 2006, 37, 106-111. [CrossRef]

18. Rashedi, E.; Nezamabadi-Pour, H.; Saryazdi, S. GSA: A gravitational search algorithm. Inf. Sci. 2009, 179, 2232-2248. [CrossRef]

19. Kaveh, A.; Talatahari, S. A novel heuristic optimization method: Charged system search. Acta Mech. 2010, 213, 267-289. [CrossRef]

20. Alatas, B. ACROA: Artificial chemical reaction optimization algorithm for global optimization. Expert Syst. Appl. 2011, 38, 13170-13180. [CrossRef]

21. Kaveh, A.; Mahdavi, V.R. Colliding bodies optimization: A novel meta-heuristic method. Comput. Struct. 2014, 139, 18-27. [CrossRef]

22. Talatahari, S.; Azizi, M. Chaos Game Optimization: A novel metaheuristic algorithm. Artif. Intell. Rev. 2020, 22, 1-88. [CrossRef]

23. Talatahari, S.; Azizi, M. Optimization of Constrained Mathematical and Engineering Design Problems Using Chaos Game Optimization. Comput. Ind. Eng. 2020, 145, 106560. [CrossRef]

24. Azizi, M. Atomic orbital search: A novel metaheuristic algorithm. Appl. Math. Model. 2021, 93, 657-683. [CrossRef]

25. Atashpaz-Gargari, E.; Lucas, C. Imperialist competitive algorithm: An algorithm for optimization inspired by imperialistic competition. In Proceedings of the 2007 IEEE Congress on Evolutionary Computation, Singapore, 25-28 September 2007; pp. 4661-4667.

26. Yang, X.S.; Deb, S. Cuckoo search via Lévy flights. In Proceedings of the 2009 World Congress on Nature \& Biologically Inspired Computing (NaBIC), Coimbatore, India, 9-11 December 2009; pp. 210-214.

27. Talatahari, S.; Azizi, M. Tribe-charged system search for global optimization. Appl. Math. Model. 2021, 93, 115-133. [CrossRef]

28. Carbas, S. Design optimization of steel frames using an enhanced firefly algorithm. Eng. Optim. 2016, 48, 2007-2025. [CrossRef]

29. Hasançebi, O.; Çarbaş, S.; Saka, M.P. Improving the performance of simulated annealing in structural optimization. Struct. Multidiscip. Optim. 2010, 41, 189-203. [CrossRef]

30. Azad, S.K. Design optimization of real-size steel frames using monitored convergence curve. Struct. Multidiscip. Optim. 2021, 63, 267-288. [CrossRef]

31. Akış, T.; Azad, S.K. Structural Design Optimization of Multi-layer Spherical Pressure Vessels: A Metaheuristic Approach. Iran. J. Sci. Technol. Trans. Mech. Eng. 2019, 43, 75-90. [CrossRef]

32. Tubishat, M.; Idris, N.; Shuib, L.; Abushariah, M.A.; Mirjalili, S. Improved Salp Swarm Algorithm based on opposition based learning and novel local search algorithm for feature selection. Expert Syst. Appl. 2020, 145, 113-122. [CrossRef]

33. Mokeddem, D.; Mirjalili, S. Improved Whale Optimization Algorithm applied to design PID plus second-order derivative controller for automatic voltage regulator system. J. Chin. Inst. Eng. 2020, 43, 541-552. [CrossRef]

34. Kaveh, A.; Hosseini, S.M.; Zaerreza, A. Improved Shuffled Jaya algorithm for sizing optimization of skeletal structures with discrete variables. In Structures; Elsevier: Amsterdam, The Netherlands, 2021; pp. 107-128. 
35. Ebrahimi, B.; Tavana, M.; Toloo, M.; Charles, V. A novel mixed binary linear DEA model for ranking decision-making units with preference information. Comput. Ind. Eng. 2020, 149, 106720. [CrossRef]

36. Azizi, M.; Ghasemi, S.A.; Ejlali, R.E.; Talatahari, S. Optimization of Fuzzy Controller for Nonlinear Buildings with Improved Charged System Search. Struct. Eng. Mech. 2020, 76, 781.

37. Wu, G.; Mallipeddi, R.; Suganthan, P.N. Problem Definitions and Evaluation Criteria for the CEC 2017 Competition on Constrained Real-Parameter Optimization; Technical Report; National University of Defense Technology: Changsha, China; Kyungpook National University: Daegu, Korea; Nanyang Technological University: Singapore, 2017.

38. Tvrdík, J.; Poláková, R. Simple framework for constrained problems with application of L-SHADE44 and IDE. In Proceedings of the 2017 IEEE Congress on Evolutionary Computation (CEC), Donostia, Spain, 5 June 2017; pp. 1436-1443.

39. Polakova, R. L-SHADE with competing strategies applied to constrained optimization. In Proceedings of the 2017 IEEE Congress on Evolutionary Computation (CEC), Donostia, Spain, 5 June 2017; pp. 1683-1689.

40. Zamuda, A. Adaptive constraint handling and success history differential evolution for CEC 2017 constrained real-parameter optimization. In Proceedings of the 2017 IEEE Congress on Evolutionary Computation (CEC), Donostia, Spain, 5 June 2017; pp. 2443-2450.

41. Derrac, J.; García, S.; Molina, D.; Herrera, F. A practical tutorial on the use of nonparametric statistical tests as a methodology for comparing evolutionary and swarm intelligence algorithms. Swarm Evol. Comput. 2011, 1, 3-18. [CrossRef]

42. Sörensen, K. Metaheuristics-The metaphor exposed. Int. Trans. Oper. Res. 2015, 22, 3-18. [CrossRef]

43. Kumar, A.; Wu, G.; Ali, M.Z.; Mallipeddi, R.; Suganthan, P.N.; Das, S. A test-suite of non-convex constrained optimization problems from the real-world and some baseline results. Swarm Evol. Comput. 2020, 56, 100693. [CrossRef]

44. Yildiz, A.R.; Abderazek, H.; Mirjalili, S. A Comparative Study of Recent Non-traditional Methods for Mechanical Design Optimization. Arch. Comput. Methods Eng. 2020, 27, 1031-1048. [CrossRef]

45. Gandomi, A.H.; Yang, X.S.; Alavi, A.H.; Talatahari, S. Bat algorithm for constrained optimization tasks. Neural Comput. Appl. 2013, 22, 1239-1255. [CrossRef]

46. Gandomi, A.H.; Yang, X.S.; Alavi, A.H. Cuckoo search algorithm: A metaheuristic approach to solve structural optimization problems. Eng. Comput. 2013, 29, 17-35. [CrossRef]

47. Ferreira, M.P.; Rocha, M.L.; Neto, A.J.; Sacco, W.F. A constrained ITGO heuristic applied to engineering optimization. Expert Syst. Appl. 2018, 110, 106-124. [CrossRef]

48. Savsani, P.; Savsani, V. Passing vehicle search (PVS): A novel metaheuristic algorithm. Appl. Math. Model. 2016, 40, 3951-3978. [CrossRef]

49. Rao, S.S. Engineering Optimization: Theory and Practice; John Wiley \& Sons: Hoboken, NJ, USA, 2019.

50. Rao, R.V.; Savsani, V.J.; Vakharia, D.P. Teaching-learning-based optimization: A novel method for constrained mechanical design optimization problems. Comput. Aided Des. 2011, 43, 303-315. [CrossRef]

51. Yu, Z.; Xu, T.; Cheng, P.; Zuo, W.; Liu, X.; Yoshino, T. Optimal Design of Truss Structures with Frequency Constraints Using Interior Point Trust Region Method. Proc. Rom. Acad. Ser. 2014, 15, 165-173.

52. Gupta, S.; Tiwari, R.; Nair, S.B. Multi-objective design optimisation of rolling bearings using genetic algorithms. Mech. Mach. Theory 2007, 42, 1418-1443. [CrossRef]

53. Zelinka, I.; Lampinen, J. Mechanical Engineering Problem Optimization by SOMA. In New Optimization Techniques in Engineering; Springer: Berlin/Heidelberg, Germany, 2004; pp. 633-653.

54. Mezura-Montes, E.; Coello, C.C.; Landa-Becerra, R. Engineering Optimization Using Simple Evolutionary Algorithm. In Proceedings of the 15th IEEE International Conference on Tools with Artificial Intelligence, Sacramento, CA, USA, 5 November 2003; pp. 149-156.

55. Akhtar, S.; Tai, K.; Ray, T. A socio-behavioural simulation model for engineering design optimization. Eng. Optim. 2002, 34, 341-354. [CrossRef]

56. Zhang, M.; Luo, W.; Wang, X. Differential evolution with dynamic stochastic selection for constrained optimization. Inf. Sci. 2008, 178, 3043-3074. [CrossRef]

57. Coello, C.A. Use of a self-adaptive penalty approach for engineering optimization problems. Comput. Ind. 2000, 41, 113-127. [CrossRef]

58. Ray, T.; Liew, K.M. Society and civilization: An optimization algorithm based on the simulation of social behavior. IEEE Trans. Evol. Comput. 2003, 7, 386-396. [CrossRef]

59. Han, J.; Yang, C.; Zhou, X.; Gui, W. A two-stage state transition algorithm for constrained engineering optimization problems. Int. J. Control Autom. Syst. 2018, 16, 522-534. [CrossRef]

60. He, Q.; Wang, L. An effective co-evolutionary particle swarm optimization for constrained engineering design problems. Eng. Appl. Artif. Intell. 2007, 20, 89-99. [CrossRef]

61. Dos Santos Coelho, L. Gaussian quantum-behaved particle swarm optimization approaches for constrained engineering design problems. Expert Syst. Appl. 2010, 37, 1676-1683. [CrossRef]

62. Zahara, E.; Kao, Y.T. Hybrid Nelder-Mead simplex search and particle swarm optimization for constrained engineering design problems. Expert Syst. Appl. 2009, 36, 3880-3886. [CrossRef]

63. Sadollah, A.; Bahreininejad, A.; Eskandar, H.; Hamdi, M. Mine blast algorithm: A new population based algorithm for solving constrained engineering optimization problems. Appl. Soft Comput. 2013, 13, 2592-2612. [CrossRef] 
64. Huang, F.Z.; Wang, L.; He, Q. An effective co-evolutionary differential evolution for constrained optimization. Appl. Math. Comput. 2007, 186, 340-356. [CrossRef]

65. Eskandar, H.; Sadollah, A.; Bahreininejad, A.; Hamdi, M. Water cycle algorithm-A novel metaheuristic optimization method for solving constrained engineering optimization problems. Comput. Struct. 2012, 110, 151-166. [CrossRef]

66. Guedria, N.B. Improved accelerated PSO algorithm for mechanical engineering optimization problems. Appl. Soft Comput. 2016, 40, 455-467. [CrossRef]

67. Garg, H. A hybrid GSA-GA algorithm for constrained optimization problems. Inf. Sci. 2019, 478, 499-523. [CrossRef]

68. Deb, K.; Srinivasan, A. Innovization: Innovating Design Principles through Optimization. In Proceedings of the 8th Annual Conference on Genetic and Evolutionary Computation, Seattle, WA, USA, 8 July 2006; pp. 1629-1636.

69. Rao, R.V.; Savsani, V.J. Mechanical Design Optimization Using Advanced Optimization Techniques; Springer Science \& Business Media: London, UK, 2012.

70. Zhang, J.; Xiao, M.; Gao, L.; Pan, Q. Queuing search algorithm: A novel metaheuristic algorithm for solving engineering optimization problems. Appl. Math. Model. 2018, 63, 464-490. [CrossRef]

71. Siddall, J.N. Optimal Engineering Design: Principles and Applications; CRC Press: London, UK, 1982.

72. Deb, K.; Goyal, M. Optimizing Engineering Designs Using a Combined Genetic Search. InICGA 1997, 521-528.

73. Coello, C.A. The Use of a Multiobjective Optimization Technique to Handle Constraints. In Proceedings of the Second International Symposium on Artificial Intelligence (Adaptive Systems); Institute of Cybernetics, Mathematics and Physics, Ministry of Science Technology and Environment: La Habana, Cuba, 1999; pp. 251-256.

74. Şahin, İ.; Dörterler, M.; Gokce, H. Optimization of Hydrostatic Thrust Bearing Using Enhanced Grey Wolf Optimizer. Mechanics 2019, 25, 480-486. [CrossRef]

75. Rao, R.V.; Waghmare, G.G. A new optimization algorithm for solving complex constrained design optimization problems. Eng. Optim. 2017, 49, 60-83. [CrossRef]

76. Lamberti, L.; Pappalettere, C. Move limits definition in structural optimization with sequential linear programming. Part I: Optimization algorithm. Comput. Struct. 2003, 81, 197-213. [CrossRef]

77. Baghlani, A.; Makiabadi, M.H. Teaching-learning-based optimization algorithm for shape and size optimization of truss structures with dynamic frequency constraints. Iran. J. Sci. Technol. Trans. Civ. Eng. 2013, 37, 409.

78. Kaveh, A.; Zolghadr, A. Shape and size optimization of truss structures with frequency constraints using enhanced charged system search algorithm. Asian J. Civ. Eng. Build. Hous. 2011, 12, 487-509.

79. Loh, H.T.; Papalambros, P.Y. Computational implementation and tests of a sequential linearization algorithm for mixed-discrete nonlinear design optimization. J. Mech. Des. 1990, 5213, 11-12. [CrossRef]

80. Kannan, B.K.; Kramer, S.N. An augmented Lagrange multiplier based method for mixed integer discrete continuous optimization and its applications to mechanical design. J. Mech. Des. 1994, 116, 405-411. [CrossRef]

81. Sandgren, E. Nonlinear integer and discrete programming in mechanical design. J. Mech. Des. 1988, 112, 223-229. [CrossRef]

82. Wang, G.G.; Hossein Gandomi, A.; Hossein Alavi, A. A chaotic particle-swarm krill herd algorithm for global numerical optimization. Kybernetes 2013, 42, 962-978. [CrossRef]

83. Wang, G.G. Adaptive response surface method using inherited latin hypercube design points. J. Mech. Des. 2003, 125, 210-220. [CrossRef] 